\title{
3. RARE EARTH ELEMENT CONSTRAINTS ON THE ORIGIN OF AMPHIBOLE IN GABBROIC ROCKS FROM SITE 894, HESS DEEP ${ }^{1}$
}

\author{
Kathryn M. Gillis²
}

\begin{abstract}
The origin of amphibole in a sequence of gabbronoritic rocks recovered at Ocean Drilling Program Site 894 was evaluated on the basis of textural and geochemical criteria. Major element compositions indicate that all the amphibole is metamorphic whereas textural relations suggest that amphibole formed either by magmatic or metamorphic processes. The rare earth element (REE) content of amphibole, clinopyroxene, apatite, and zircon were determined by ion microprobe to assess these interpretations. Amphibole shows broad range in REE concentrations (up to 1,000 times chondritic values) with $(\mathrm{La} / \mathrm{Sm})_{N}$ ratios from 0.1 to 1.4. There is no systematic relationship between REE content and textural type. The origin of amphibole with REE contents less than or equal to clinopyroxene cannot be distinguished by compositional criteria. By contrast, the REE content of all other amphibole can only be explained by magmatic processes. Calculated melts in equilibrium with igneous amphibole indicate that amphibole crystallized from melts with progressively higher REE contents; in some samples melts evolved without significant fractionation of the REEs whereas in other samples melts became light-REE-enriched. An increase in the total abundance of the REE melts is consistent with the crystallization of plagioclase, clinopyroxene, and orthopyroxene from progressively more evolved melt. The light-REE-enriched melts may have been influenced by the crystallization of accessory phases or by interaction with exsolved magmatic fluids. These results indicate that there was a continuum between magmatic and hydrothermal processes and that groundmass amphibole records a complex history of crystallization from a melt and its exsolved magmatic fluids, followed by interaction with seawater-derived hydrothermal fluids.
\end{abstract}

\section{INTRODUCTION}

Amphibole may form as a late-stage magmatic phase, crystallizing from chemically-evolved late-stage melts or exsolved magmatic fluids as well as a hydrothermal phase replacing pyroxene and olivine or filling fractures in oceanic plutonics (e.g., Mével, 1987). Distinction between these origins has been evaluated using textural relationships, major and minor element compositions, and stable isotopic compositions (e.g., Stakes et al., 1991). In some cases, determination of the origin of amphibole is straightforward using a combination of these criteria. For example, brown, high- $\mathrm{TiO}_{2}(3-4 \mathrm{wt} \%)$, pargasitic amphibole that forms coronitic overgrowths around clinopyroxene is considered to be magmatic whereas green, low- $\mathrm{TiO}_{2}(<0.5 \mathrm{wt} \%)$ pargasitic amphibole that fills fractures in mylonitic zones is considered hydrothermal (e.g., Mével and Cannat, 1991). In most cases, however, the origin of amphibole is ambiguous.

Development of reliable criteria to determine the origin of amphibole is critical to our understanding of the magma-hydrothermal transition in oceanic hydrothermal systems. Characterizing the distribution and abundance of magmatic amphibole will place important constraints on the evolution of late-stage melts and magmatic fluids in oceanic plutonics. Moreover, determining the distribution of hydrothermal amphibole is essential as this phase typically records the conditions at which hydrothermal fluids first penetrate the lower crust.

During Ocean Drilling Program (ODP) Leg 147, two sites were drilled within the Cocos-Nazca rift valley at Hess Deep to investigate the evolution of crustal processes at the fast-spreading East Pacific Rise (EPR). At Site 894, a sequence of high-level gabbroic rocks was recovered in a series of seven holes that were drilled at the western end of an intrarift ridge in $\sim 1$ Ma crust. Amphibole is a common

'Mével, C., Gillis, K.M., Allan, J.F., and Meyer, P.S. (Eds.), 1996. Proc. ODP, Sci. Results, 147: College Station, TX (Ocean Drilling Program).

${ }^{2}$ School of Earth and Ocean Sciences, University of Victoria, P.O. Box 1700, Victoria, British Columbia, Canada V8W 2Y2. kgillis@postoffice.uvic.ca phase in all lithologic units of Hole $894 \mathrm{G}$, in the form of isolated grains intersertal to plagioclase, rims on pyroxene, and fracture infillings. Although these textures may suggest that the amphibole formed either by magmatic or metamorphic processes, the major compositions of all textural types indicate a metamorphic origin. To further investigate the origin of amphibole, I have determined the rare earth element (REE) content of amphibole with a variety of textures in samples representative of all lithologic types and grain size. I will show that the composition of amphibole records a complex evolution that involved chemically evolved-melts, exsolved magmatic fluids, and seawater-derived hydrothermal fluids.

\section{IGNEOUS LITHOSTRATIGRAPHY AND METAMORPHIC CHARACTERISTICS OF HOLE 894G}

Hole $894 \mathrm{G}$ penetrated 154.5 meters below seafloor (mbsf), with an average recovery of $35.4 \%$. The igneous rock types recovered include gabbronorite, olivine gabbronorite, gabbro, olivine gabbro, $\mathrm{Fe}$ Ti oxide gabbronorite, and basalt, in order of their abundance (Shipboard Scientific Party, 1993). Thirteen igneous lithostratigraphic units were recognized on the basis of mineralogy, texture, and grain size (Shipboard Scientific Party, 1993). Variation in texture and mineralogy is common on a thin section and hand specimen scale such that equigranular gabbronorite occurs within poikiolitic gabbronorite and coarse-grained gabbro. Between 100 and 120 mbsf, a magmatic foliation, defined by the alignment of tabular plagioclase grains, occurs within fine-grained gabbro and, less commonly, coarser grained gabbronorite. Apatite and zircon locally occur in trace amounts $(<0.1$ modal\%), associated with $\mathrm{Fe}-\mathrm{Ti}$ oxides and brownish green amphibole in coarse-grained patches of gabbro and gabbronorite. The anorthite content of plagioclase (An) ranges from 50 to 70 , although a few samples are more calcic with An contents up to 95 (Natland and Dick, this volume; Pedersen et al., this volume). The $\mathrm{Mg} \#\left(\mathrm{Mg} / \mathrm{Mg}+\mathrm{Fe}^{2+}\right)$ of clinopyroxene and orthopyroxene range from 62 to 80 and from 63 
to 73, respectively (Natland and Dick, this volume; Pedersen et al., this volume). The variation in texture and grain size and the presence of evolved rock types in the Site 894 plutonics are similar to high level plutonics in many ophiolites (e.g., Pedersen, 1986).

The gabbroic rocks recovered at Hole $894 \mathrm{G}$ are variably altered to amphibolite to zeolite facies assemblages (Shipboard Scientific Party, 1993; Früh-Green, this volume; Lécuyer and Gruau, this volume; Manning and MacLeod, this volume). Higher temperature assemblages are commonly overprinted by lower temperature assemblages and there is no systematic variation in either metamorphic grade or degree of alteration with depth. Secondary minerals replace $20 \%-$ $50 \%$ of the primary phases in most of the drillcore, except adjacent to macroscopic fractures or cataclastic zones where $50 \%-80 \%$ of the groundmass phases are replaced. Several generations of veins crosscut the gabbroic rocks, ranging from microfractures filled with amphibole to macroscopic fractures filled with assemblages of amphibole, chlorite, prehnite, epidote, smectite, calcite, and zeolites.

\section{METHODS, SAMPLE SELECTION, AND ANALYTICAL STRATEGY}

Mineral compositions were determined by wavelength dispersive techniques using a JEOL 733 electron microprobe equipped with Tracor Northern software at Massachusetts Institute of Technology (MIT); standard Bence-Albee corrections and natural mineral standards were used. Data collection was guided by backscattered electron images.

Rare earth element concentrations were measured using a Cameca IMS $3 \mathrm{f}$ ion microprobe at Woods Hole Oceanographic Institution. Operating conditions were similar to those reported by Shimizu and Le Reox (1986) and Johnson et al. (1990). Concentrations were determined using empirical relationships between secondary ion intensity (relative to $\mathrm{Si}$ or $\mathrm{Ca}$ ) and concentration (working curves) established for mineral standards. Trace element abundances for clinopyroxene were calculated from intensity values, ratioed to ${ }^{30} \mathrm{Si}$, using working curves developed by Shimizu and Le Reox (1986). Interferences of barium oxides and hydroxides with ${ }^{151} \mathrm{Eu}$ and ${ }^{153} \mathrm{Eu}$ were considered to be negligible as $\mathrm{Ba}$ is highly incompatible in clinopyroxene (Johnson and Dick, 1992). Trace element abundances for amphibole were calculated using working curves developed by Sisson (1994). Because interferences of barium oxides and hydroxides with ${ }^{151} \mathrm{Eu}$ and ${ }^{153} \mathrm{Eu}$ are likely in amphibole, there is no correction factor for $\mathrm{Eu}$; inconsistent data between analytical sessions precluded a correction factor for $\mathrm{Yb}$ (Sisson, 1994). In the absence of working curves for zircon, REE concentrations were calculated using the clinopyroxene working curves. Trace element abundances for apatite were calculated from intensity values, ratioed to ${ }^{44} \mathrm{Ca}$, using correction factors derived by Grandjean (1989). The spot size was $\sim 15-20 \mathrm{~mm}$ and the accuracy and precision for clinopyroxene, amphibole, and apatite are believed to be $\sim 10 \%-20 \%$ for the light REE, and $\sim 10 \%-15 \%$ for the middle and heavy REE. The accuracy of the zircon analyses is unknown.

Ten samples were selected from Hole $894 \mathrm{G}$ that spanned the range in rock type, grain size, and depth. The igneous characteristics of these samples are given in Table 1 . The major element composition of the primary minerals in these samples indicate that they are slightly more chemically evolved than the average composition for Hole $894 \mathrm{G}$ (see Natland and Dick, this volume). Amphibole grains within these samples were selected for ion probe analysis if their textures or major and minor element compositions suggested a magmatic or high temperature hydrothermal origin. Amphibole that filled fractures was avoided. Clinopyroxene grains were selected for ion probe analysis to provide a comparison to amphibole or because its texture and/or composition indicated a secondary origin.

Many of the trace element analyses reported in Table 2 reflect the composition of more than one mineral phase due to fine-grained in- tergrowths of hydrothermal minerals. REE analyses for amphibole generally represent zones within a grain with a uniform major element composition, but a few analyses included a mixture of high- and low- $\mathrm{Al}_{2} \mathrm{O}_{3}$ amphibole. Clinopyroxene $\mathrm{REE}$ analyses may include clinopyroxene, exsolution lamellae of orthopyroxene, and minor amphibole. The proportion of these phases for each analysis are reported in the Appendix.

\section{RESULTS}

\section{Clinopyroxene}

\section{Textural Relations}

Clinopyroxene in the Hole $894 \mathrm{G}$ gabbroic rocks occurs as oikocrysts and equigranular grains (Fig. 1A, C). It is variably altered to assemblages of amphibole, magnetite, and secondary clinopyroxene, with incipient alteration to amphibole being focused along grain boundaries and cleavages. Secondary clinopyroxene forms as isolated blebs that lack exsolution textures within its magmatic precursor or along grain boundaries. It typically has higher birefingence in comparison to its precursor, and locally contains fluid inclusions trapped during mineral growth.

\section{Major and Minor Element Compositions}

Clinopyroxene has a narrow range in $\mathrm{Mg \#}(0.68-0.75)$ and shows considerable scatter in $\mathrm{Na}_{2} \mathrm{O}(0.05-0.42 \mathrm{wt} \%), \mathrm{TiO}_{2}(0.1-1.0 \mathrm{wt} \%)$, $\mathrm{Al}_{2} \mathrm{O}_{3}(0.1-2.5 \mathrm{wt} \%), \mathrm{MnO}(0.05-0.50 \mathrm{wt} \%)$, and $\mathrm{Cr}_{2} \mathrm{O}_{3}(0.05-0.40$ $\mathrm{wt} \%$ ) content (Table 3 ). The wollanstonite component of an individual grain may vary by $5-8 \mathrm{~mol} \%$ without change in $\mathrm{Mg} \#$ and minor element content. The minor elements, $\mathrm{Na}_{2} \mathrm{O}, \mathrm{TiO}_{2}, \mathrm{Al}_{2} \mathrm{O}_{3}$, and $\mathrm{Cr}_{2} \mathrm{O}_{3}$, however, decrease sharply in concentration at wollanstonite $\left(\mathrm{CaSiO}_{3}\right)$ contents $>43$ to $44 \mathrm{~mol} \%$. Where textural evidence for exsolution (i.e., presence of orthopyroxene lamellae in clinopyroxene) was observed using backscattered electron images, the wollanstonite contents were $<44 \mathrm{~mol} \%$, however, these textural relations were often obscured by the alteration of clinopyroxene to amphibole. Based on these textural and compositional criteria, the transition from magmatic to secondary clinopyroxene is estimated at wollanstonite contents of 43-44 mol\%, similar to other plutonic suites (Manning and Bird, 1986; Stakes et al., 1991).

\section{REE Contents}

Clinopyroxene has a narrow range in REE content and chondritenormalized profiles (Table 2; Fig. 2). The REE content of clinopyroxene typically ranges from 1 to 10 times and from 10 to 30 times the chondrite-normalized values for light and heavy REE, respectively. All clinopyroxene grains are light-REE-depleted with flat heavy $\mathrm{REE} ;(\mathrm{La} / \mathrm{Sm})_{\mathrm{N}}$ and $(\mathrm{Dy} / \mathrm{Er})_{\mathrm{N}}$ ratios range from 0.05 to 0.4 and from 0.95 to 1.4 , respectively (Fig. 2). Individual grains are generally homogeneous in composition; however, most analyses were collected away from grain boundaries as these zones are preferentially altered.

A few clinopyroxene grains in Samples 147-894G-17R-1, 81-86 $\mathrm{cm}$ (Piece 11), and 18R-1, 2-3 cm (Piece 1), have wollastonite contents $>44 \mathrm{~mol} \%$ and are interpreted to be secondary in origin. These grains do not show any systematic trends in their REE content. For example, in Sample 147-894G-18R-1, 2-3 cm (Piece 1), the REE abundances in secondary clinopyroxene are both depleted and similar to magmatic clinopyroxene (cf. analyses 6 and 8; Fig. 2P).

\section{Amphibole}

\section{Textural Relations}

Amphibole is a minor phase in all rock types throughout Hole $894 \mathrm{G}$, both in the groundmass and filling micro- and macroscopic 
Table 1. Summary of igneous characteristics of analyzed samples, Hole 894G.

\begin{tabular}{|c|c|c|c|c|c|c|c|c|c|c|}
\hline $\begin{array}{l}\text { Core, section, } \\
\text { interval }(\mathrm{cm})\end{array}$ & $\begin{array}{l}\text { Piece } \\
\text { no. }\end{array}$ & Lithology & $\begin{array}{l}\text { Unit } \\
\text { no. }\end{array}$ & $\begin{array}{l}\text { Depth } \\
\text { (mbsf) }\end{array}$ & $\begin{array}{l}\text { Grain } \\
\text { size }\end{array}$ & Igneous texture & $\begin{array}{l}\text { Plag } \\
\text { (An) }\end{array}$ & $\begin{array}{c}\mathrm{Cpx} \\
(\mathrm{Mg \# )}\end{array}$ & $\begin{array}{c}\text { Opx } \\
(\mathrm{Mg} \#)\end{array}$ & Accessory phases \\
\hline \multicolumn{11}{|l|}{ 147-894G- } \\
\hline $6 \mathrm{R}-1,112-116$ & 10 & Gabbronorite & 6 & 55.92 & $\mathrm{~m}$ & Granular-intergranular & 0.53 & 0.70 & - & Fe-Ti oxides \\
\hline $8 R-1,13-15$ & 2 & Gabbronorite & 6 & 68.63 & f to $m$ & Poikiolitic & 0.56 & 0.72 & 0.70 & Fe-Ti oxides, apatite \\
\hline $9 \mathrm{R}-3,51-54$ & 4 & Gabbro & 6 & 77.59 & $\mathrm{~m}$ to $\mathrm{c}$ & Intergranular & 0.48 & - & - & Fe-Ti oxides, apatite \\
\hline $9 R-3,70-76$ & $5 \mathrm{C}$ & Oxide gabbronorite & 6 & 77.78 & $\mathrm{~m}$ to $\mathrm{c}$ & Intergranular-pegmatitic & 0.48 & - & - & $\mathrm{Fe}-\mathrm{Ti}$ oxides, zircon \\
\hline $12 \mathrm{R}-1,117-125$ & 12D & Gabbro & 11 & 94.97 & $\mathrm{~m}$ to $\mathrm{c}$ & Granular-intergranular & 0.59 & 0.72 & - & Fe-Ti oxides, apatite \\
\hline $12 \mathrm{R}-3,112-115$ & $7 \mathrm{~A}$ & Gabbronorite & 11 & 97.85 & $\mathrm{~m}$ to $\mathrm{c}$ & Granular-intergranular & 0.53 & 0.72 & 0.69 & Fe-Ti oxides \\
\hline $12 \mathrm{R}-5,50-57$ & $7 \mathrm{~B}$ & Gabbro & 11 & 100.16 & f to c & Intergranular & 0.54 & 0.71 & - & Fe-Ti oxides \\
\hline $17 \mathrm{R}-1,23-25$ & 6 & Gabbro & 11 & 126.03 & $f$ to $c$ & Pegmatitic & 0.51 & - & - & Fe-Ti oxides, apatite \\
\hline $17 R-1,81-86$ & 11 & Gabbronorite & 11 & 126.61 & f to $m$ & Granular-poikiolitic & 0.52 & 0.71 & 0.68 & Fe-Ti oxides \\
\hline $18 \mathrm{R}-1,2-3$ & 1 & Gabbronorite & 11 & 130.92 & f to $\mathrm{m}$ & Granular-poikiolitic & 0.58 & 0.75 & - & Fe-Ti oxides \\
\hline
\end{tabular}

Notes: Depths are curated depths. Grain sizes are as follows: $f=$ fine $(<1 \mathrm{~mm}), \mathrm{m}=$ medium $(1-5 \mathrm{~mm})$, and $\mathrm{c}=\mathrm{coarse}(5-30 \mathrm{~mm})$. See Shipboard Scientific Party $(1993)$ for definition of textures and unit numbers. Plag $(\mathrm{An})=$ average composition of primary plagioclase. $\mathrm{Cpx}(\mathrm{Mg} \#)=$ average composition of clinopyroxene with wollastonite content $<44$ mol\%. Dashes $(-)=$ not determined.

fractures (Shipboard Scientific Party, 1993). The most common occurrence of amphibole in the groundmass rims clinopyroxene in the form of fibrous, green grains (Fig. 1A) or granular, brownish green grains that are optically zoned (Fig. 1C). Fibrous, green amphibole rims generally have a preferred orientation that parallel exsolution lamellae, and are intergrown with very fine-grained, disseminated magnetite and relict clinopyroxene, suggesting that amphibole replaced igneous clinopyroxene. Granular, brownish green amphibole rims on clinopyroxene are locally intergrown with $\mathrm{Fe}-\mathrm{Ti}$ oxides and may be partly replaced by fibrous green amphibole. Backscattered images show that granular rims are composed of amphibole and that relict clinopyroxene and disseminated magnetite only occur immediately adjacent to clinopyroxene, suggesting that granular amphibole only partly replaced igneous clinopyroxene. Groundmass amphibole also forms as isolated euhedral grains (Fig. 1B) or clots of randomly oriented grains (Fig. ID) that are intersertal to plagioclase or adjacent to Fe-Ti oxides; these grains may be partly replaced by fibrous green amphibole. This brownish green to green amphibole is most common in gabbro intervals and coarse-grained patches of gabbro within gabbronorite. Backscattered images show that isolated amphibole grains are composed of amphibole with a range of composition but do not contain relict clinopyroxene or disseminated magnetite, suggesting that they do not replace primary minerals. Samples containing these amphiboles have slightly more evolved primary mineral compositions than the Hole 894G average (Natland and Dick, this volume); coarse-grained patches that host accessory phases are the most evolved of all (Table 1). Textural relations suggest that the onset of amphibole crystallization postdated plagioclase, clinopyroxene, and orthopyroxene, may or may not have been coeval with apatite, and preceded zircon.

\section{Major and Minor Element Compositions}

Most of the amphibole in the gabbroic rocks of Hole $894 \mathrm{G}$ is calcic, with a range in composition from actinolite to magnesio-hornblende (nomenclature after Leake, 1978). All amphibole grains have the following characteristics: (1) $\mathrm{CaO}$ contents increase systematically with increasing $\mathrm{FeO}$ and have a weak negative correlation with $\mathrm{MgO}$; (2) $\mathrm{MnO}$ contents are generally $<0.4 \mathrm{wt} \%$ with a few grains up to 1.4 wt \% ; (3) $\mathrm{Cr}_{2} \mathrm{O}_{3}$ contents are $<0.15$ to 0.35 wt \% ; (4) $\mathrm{K}_{2} \mathrm{O}$ contents are $<0.6 \mathrm{wt} \%$; and (5) $\mathrm{F}$ and $\mathrm{Cl}$ contents are $<0.6$ and $<0.3 \mathrm{wt} \%$, respectively (Table 4 ). The $\mathrm{Al}^{\mathrm{IV}}$ content of all occurrences systematically increases with Site A occupancy and Ti content and falls within the compositional range of other samples from Hole $894 \mathrm{G}$ and Hess Deep (Fig. 3). These compositions indicate a metamorphic origin at greenschist to the transition to the amphibolite facies conditions (Liou et al., 1974).

\section{REE Contents}

Amphibole has a broad range in REE profiles. $(\mathrm{La} / \mathrm{Sm})_{\mathrm{N}}$ and $(\mathrm{Dy} / \mathrm{Er})_{\mathrm{N}}$ ratios range from 0.1 to 1.4 and from 0.08 to 1.6 , respectively, and concentrations may vary within an individual sample by 1000 times chondritic values (Fig. 2; Table 2). Two compositional groups are distinguished by the abundance and shape of their REE profile. One group has REE profiles that mimic those of clinopyroxene with REE concentrations that are slightly depleted or enriched relative to clinopyroxene; $(\mathrm{La} / \mathrm{Sm})_{\mathrm{N}}$ and $(\mathrm{Dy} / \mathrm{Er})_{\mathrm{N}}$ ratios are $<0.4$ and $>1.3$, respectively (Figs. 2, 4A). Another group of amphiboles are enriched in light REE relative to clinopyroxene with $(\mathrm{La} / \mathrm{Sm})_{\mathrm{N}}$ ratios $>0.4$ to 1.4; concentrations are similar to the light-REE-depleted amphibole. Light-REE-depleted and -enriched amphibole cannot be distinguished on the basis of major and minor element compositions or textural types.

Individual amphibole grains may be homogeneous or show a broad range in REE content. In Sample 147-894G-6R-1, 112-116 cm (Piece 10), the core of a granular, isolated grain (analyses 6 and 7; Fig. 2A; Table 2) is more enriched in REEs but is less aluminous than its fibrous rim (analysis 8). In Sample 147-894G-12R-1, 117-125 cm (Piece 12D), the core of an amphibole grain rimming clinopyroxene is light-REE-enriched (analyses 1,2, and 6), whereas toward the rim it is light-REE-depleted (analyses 3 and 16). There is no systematic variation in the major element composition of this grain (see Appendix). In Sample 147-894G-12R-3, 112-115 cm (Piece 7A), an amphibole grain rimming clinopyroxene is both light-REE-enriched and aluminous (analyses 5-7) and light-REE-depleted and more actinolitic (analysis 8; Table 2; Fig. 2I) but without a systematic zonation. In Sample 147-894G-12R-5, 50-59 cm (Piece 7B), the core of an isolated grain has a REE profile that mimics clinopyroxene (analysis 1; Table 2; Fig. 2J) whereas the rim is enriched in middle to heavy REE (analysis 2; Table 2; Fig. 2J). There is no zonation in major and minor element composition.

Amphibole grains within an individual sample show the same range in REE content as in individual grains. In Sample 147-894G$8 \mathrm{R}-1,13-15 \mathrm{~cm}$ (Piece 2), an amphibole grain that rims clinopyroxene and an isolated amphibole grain both have chondrite-normalized profiles (Fig. 2C) that mimic clinopyroxene (Fig. 2D). The major element composition of these grains are uniform and similar, with $\mathrm{Al}^{\mathrm{IV}}$ contents from 0.57 to 1.0 , and Mg\#s from 0.68 to 0.76 . In Sample 147-894G-9R-3, 71-76 cm (Piece 5A), two isolated amphibole grains were analyzed. One grain from this sample is surrounded by plagioclase (analysis 4 and 5), and the other grain (analyses 7 and 8) is intergrown with Fe-Ti oxides (analyses 7 and 8 occur within 100 and $500 \mu \mathrm{m}$ of the Fe-Ti oxides, respectively). Both grains have similar REE profiles, however, the grain surrounded by plagioclase is 
Table 2. Rare-earth-element data for amphibole, clinopyroxene, apatite, and zircon.

\begin{tabular}{|c|c|c|c|c|c|c|c|c|c|c|c|c|c|}
\hline $\begin{array}{c}\text { Analysis } \\
\text { no. }\end{array}$ & Mineral & Comments & $\mathrm{La}$ & $\mathrm{Ce}$ & $\mathrm{Nd}$ & $\mathrm{Sm}$ & Eu & Dy & Er & $\mathrm{Yb}$ & $\mathrm{La} / \mathrm{Sm}$ & Dy/Er & $\mathrm{La} / \mathrm{Er}$ \\
\hline \multirow{2}{*}{\multicolumn{14}{|c|}{ 147-894G-6R-1, 112-116 cm (Piece 10): }} \\
\hline & & & & 13.0 & 13.9 & 5.8 & - & 6.4 & 5.1 & - & 0.35 & 0.82 & 0.42 \\
\hline 2 & Amp & Rim clinopyroxene (3) & 3.2 & 12.6 & 11.1 & 4.8 & - & 4.8 & 3.8 & - & 0.35 & 0.82 & 0.42 \\
\hline 3 & $\mathrm{Cpx}_{\mathrm{px}}$ & 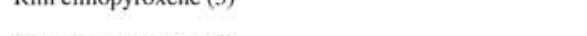 & 0.9 & 3.0 & 2.8 & 1.3 & 0.3 & 2.5 & 1.4 & 1.4 & 0.42 & 0.82 & 0.57 \\
\hline 4 & Amp & Rim clinopyroxene (3) & 3.6 & 14.3 & 14.7 & 6.0 & - & 5.9 & 4.7 & -1.4 & 0.41 & 1.18 & 0.43 \\
\hline 6 & Amp & Isolated grain, same grain as $(7,8)$ & 5.1 & 25.5 & 22.7 & 7.8 & - & 5.9 & 4.7 & - & 0.37 & 0.63 & 0.60 \\
\hline 7 & Amp & Isolated grain, same grain as $(7,8)$ & 4.7 & 21.0 & 14.1 & 3.9 & $\overline{-}$ & 3.4 & 2.7 & $\overline{-}$ & 0.41 & 0.82 & 0.74 \\
\hline 8 & Amp & Isolated grain, same grain as $(7,9)$ & 1.2 & 6.8 & 7.8 & 3.6 & $=$ & 2.8 & 2.3 & $\overline{-}$ & 0.75 & 0.82 & 1.18 \\
\hline 9 & Cpx & Same grain as $(9,10)$ & 2.0 & 9.6 & 11.7 & 6.3 & $\overline{1.2}$ & 10.5 & 5.9 & 5.9 & 0.22 & 0.82 & 0.37 \\
\hline 10 & $\mathrm{Cpx}$ & Same grain as $(9,11)$ & 1.9 & 7.4 & 7.6 & 4.1 & 1.0 & 7.2 & 4.1 & 4.4 & 0.20 & 1.15 & 0.23 \\
\hline 11 & $\mathrm{Cpx}$ & Same grain as $(9,10)$ & 2.1 & 8.7 & 9.0 & 4.8 & 1.0 & 8.5 & 4.6 & 5.1 & 0.28 & 1.13 & 0.31 \\
\hline \multicolumn{14}{|c|}{ 147-894G-8R-1, 13-15 cm (Piece 2): } \\
\hline 1 & $\mathrm{Cpx}$ & Same grain as $(2,3)$ & 1.7 & 7.8 & 9.4 & 5.0 & 1.1 & 8.5 & 4.8 & 4.6 & 0.22 & 1.16 & 0.25 \\
\hline 2 & $\mathrm{Cpx}$ & Same grain as $(1,3)$ & 1.3 & 6.3 & 7.5 & 3.8 & 0.8 & 6.4 & 3.8 & 3.5 & 0.22 & 1.11 & 0.24 \\
\hline 3 & $\mathrm{Cpx}$ & Same grain as $(1,2)$ & 1.6 & 7.0 & 8.1 & 4.2 & 0.8 & 6.4 & 4.0 & 3.9 & 0.24 & 1.04 & 0.28 \\
\hline 5 & $\mathrm{Cpx}$ & Sanie gram as $(1,2)$ & 2.3 & 9.3 & 9.1 & 4.6 & 1.1 & 7.3 & 4.7 & 4.4 & 0.31 & 1.01 & 0.32 \\
\hline 7 & Amp & Rim clinopyroxene, same grain as (8), next to apatite (12) & 1.3 & 6.8 & 7.1 & 3.1 & - & 5.6 & 2.9 & - & 0.27 & 1.26 & 0.31 \\
\hline 8 & Amp & Rim clinopyroxene, same grain as (7), next to apatite (12) & 1.2 & 5.2 & 6.4 & 3.2 & - & 5.2 & 3.0 & $=$ & 0.22 & 1.13 & 0.26 \\
\hline 10 & Amp & Isolated grain & 1.0 & 5.2 & 6.3 & 3.2 & - & 5.2 & 3.0 & - & 0.19 & 1.15 & 0.23 \\
\hline 12 & Apatite & Next to amphibole $(7,8)$ & 128.0 & 449.0 & 393.0 & 94.7 & 7.4 & 87.4 & 31.2 & 23.1 & 1.35 & 1.80 & 2.77 \\
\hline \multicolumn{14}{|c|}{ 147-894G-9R-3, 51-54 cm (Piece 4): } \\
\hline 1 & Amp & Groundmass clot & 2.5 & 16.5 & 21.8 & 10.4 & - & 20.1 & 10.5 & - & 0.15 & 1.25 & 0.16 \\
\hline 2 & Amp & Groundmass clot & 2.2 & 13.9 & 18.2 & 7.9 & - & 14.0 & 6.6 & - & 0.18 & 1.38 & 0.23 \\
\hline 3 & Amp & Groundmass clot & 1.9 & 11.6 & 15.6 & 5.7 & - & 10.1 & 4.9 & - & 0.21 & 1.35 & 0.26 \\
\hline 4 & Amp & Groundmass clot & 2.5 & 16.2 & 20.3 & 8.1 & - & 14.5 & 6.9 & - & 0.20 & 1.37 & 0.25 \\
\hline \multicolumn{14}{|c|}{ 147-894G-9R-3, 70-76 cm (Piece 5C): } \\
\hline 1 & Amp & Groundmass clot & 3.4 & 20.2 & 27.0 & 10.8 & - & 17.2 & 9.3 & - & 0.20 & 1.21 & 0.25 \\
\hline 2 & Amp & Groundmass clot & 4.9 & 32.7 & 51.8 & 21.4 & - & 30.9 & 15.4 & - & 0.14 & 1.32 & 0.22 \\
\hline 4 & Amp & Isolated grain, same grain as (5) & 1.5 & 10.0 & 15.2 & 6.8 & - & 12.7 & 6.4 & - & 0.14 & 1.30 & 0.16 \\
\hline 5 & Amp & Isolated grain, same grain as (4) & 4.4 & 23.2 & 36.5 & 17.3 & - & 31.9 & 15.1 & - & 0.16 & 1.38 & 0.20 \\
\hline 6 & Amp & Isolated grain, same grain as (7) & 56.7 & 316.0 & 313.0 & 98.6 & - & 104 & 51.6 & - & 0.36 & 1.32 & 0.74 \\
\hline 7 & Amp & Isolated grain, same grain as (8) & 6.1 & 40.0 & 57.1 & 23.2 & - & 38.5 & 17.7 & - & 0.17 & 1.42 & 0.23 \\
\hline 8 & Amp & Isolated grain & 1.5 & 11.3 & 16.7 & 7.4 & - & 15.0 & 8.1 & - & 0.13 & 1.21 & 0.13 \\
\hline 9 & Zircon & & 0.7 & 11.5 & 11.4 & 15.9 & - & 238 & 386 & 679 & 0.03 & 0.40 & 0.00 \\
\hline \multicolumn{14}{|c|}{ 147-894G-9R-3, 70-76 cm (Piece 5C): } \\
\hline 10 & Zircon & & 0.2 & 15.5 & 9.0 & 23.9 & - & 602 & 995 & 1564 & 0.01 & 0.40 & 0.00 \\
\hline 11 & Amp & Isolated grain, next to zircon & 1.4 & 9.3 & 17.2 & 8.9 & - & 18.9 & 8.2 & (150 & 0.10 & 1.51 & 0.11 \\
\hline 12 & Zircon & & 0.4 & 11.6 & 8.3 & 15.7 & - & 467 & 861 & 1373 & 0.02 & 0.36 & 0.00 \\
\hline 13 & Zircon & & 1.4 & 11.6 & 15.3 & 14.6 & - & 149 & 247 & 420 & 0.06 & 0.39 & 0.00 \\
\hline 14 & Amp & Isolated grain, next to zircon & 1.4 & 11.6 & 23.0 & 11.0 & - & 20.7 & 10.6 & - & 0.08 & 1.28 & 0.09 \\
\hline 15 & Amp & Isolated grain, next to zircon & 1.1 & 8.9 & 14.8 & 7.9 & - & 14.4 & 7.2 & - & 0.08 & 1.31 & 0.10 \\
\hline \multicolumn{14}{|c|}{ 147-894G-12R-1, 117-125 cm (Piece 12D): } \\
\hline 1 & Amp & Rim clinopyroxene, same grain as $(2,3,6,16)$ & 14.0 & 55.2 & 44.6 & 13.2 & - & 23.4 & 13.3 & - & 0.67 & 1.16 & 0.72 \\
\hline 2 & Amp & Rim clinopyroxene, same grain as $(1,3,6,16)$ & 142.0 & 612.3 & 325.2 & 67.9 & - & 95.7 & 51.0 & - & 1.31 & 1.23 & 1.89 \\
\hline 3 & Amp & Rim clinopyroxene, same grain as $(1,2,6,16)$ & 2.2 & 11.4 & 11.2 & 4.6 & - & 7.7 & 3.6 & - & 0.30 & 1.38 & 0.40 \\
\hline 6 & Amp & Rim clinopyroxene, same grain as $(1,2,316)$ & 43.8 & 198.4 & 113.4 & 28.1 & - & 36.7 & 17.8 & $\overline{-}$ & 0.98 & 1.35 & 1.67 \\
\hline 7 & Amp & $\begin{array}{l}\text { Groundmass clot }\end{array}$ & 0.7 & 5.1 & 11.1 & 6.0 & - & 14.2 & 6.6 & $=$ & 0.07 & 1.40 & 0.07 \\
\hline 9 & Amp & Isolated grain & 1.1 & 7.7 & 11.6 & 4.4 & $=$ & 7.4 & 3.1 & - & 0.16 & 1.60 & 0.26 \\
\hline 10 & Amp & Groundmass clot, same grain as $(11,12,13)$ & 3.0 & 9.5 & 8.1 & 2.4 & - & 4.4 & 2.4 & - & 0.78 & 1.20 & 0.83 \\
\hline iI & Amp & Groundmass clot, same grain as $(10,12,13)$ & 1.6 & 7.5 & 7.4 & 2.2 & - & 2.3 & 1.3 & $=$ & 0.45 & 1.18 & 0.85 \\
\hline 12 & Amp & Groundmass clot, same grain as $(10,11,13)$ & 2.0 & 7.9 & 10.6 & 3.9 & - & 7.0 & 3.8 & $\bar{z}$ & 0.32 & 1.21 & 0.36 \\
\hline 13 & Amp & Groundmass clot, same grain as $(10,11,12)$ & 2.1 & 6.7 & 6.6 & 2.7 & - & 3.8 & 2.0 & $=$ & 0.48 & 1.24 & 0.69 \\
\hline 14 & $\mathrm{Cpx}$ & Same grain as (15) & 1.5 & 6.6 & 9.2 & 4.6 & 1.1 & 8.9 & 5.1 & 5.2 & 0.21 & 1.15 & 0.21 \\
\hline 15 & $\mathrm{Cpx}$ & Same grain as (14) & 1.5 & 6.3 & 8.4 & 5.9 & 1.0 & 8.5 & 5.2 & 5.1 & 0.15 & 1.07 & 0.19 \\
\hline 16 & Amp & Rim clinopyroxene, same grain as $(1,2,3,6)$ & 7.7 & 34.5 & 29.8 & 10.5 & - & 15.5 & 6.2 & & 0.46 & 1.64 & 0.84 \\
\hline 17 & Apatite & & 241 & 935 & 909 & 233 & - & 238 & 109 & 86.8 & 0.65 & 1.40 & 2.21 \\
\hline
\end{tabular}


Table 2 (continued).

\begin{tabular}{|c|c|c|c|c|c|c|c|c|c|c|c|c|c|}
\hline $\begin{array}{c}\text { Analysis } \\
\text { no. }\end{array}$ & Mineral & Comments & $\mathrm{La}$ & $\mathrm{Ce}$ & $\mathrm{Nd}$ & Sm & Eu & Dy & Er & $\mathrm{Yb}$ & $\mathrm{La} / \mathrm{Sm}$ & Dy/Er & $\mathrm{La} / \mathrm{Er}$ \\
\hline \multicolumn{14}{|c|}{ 147-894G-12R-3, 112-115 cm (Piece 7A): } \\
\hline $2_{2}^{2}$ & & & 1.3 & 6.2 & 8.0 & 4.1 & 1.0 & 5.8 & 4.0 & 4.0 & 0.20 & 0.94 & 0.22 \\
\hline 3 & Amp & Groundmass clot & 1.5 & 7.1 & 8.5 & 4.0 & - & 7.3 & 3.4 & - & 0.23 & 1.40 & 0.30 \\
\hline 4 & Amp & Groundmass clot & 1.7 & 7.5 & 10.2 & 4.3 & - & 7.5 & 3.3 & - & 0.24 & 1.48 & 0.34 \\
\hline 5 & Amp & Rim clinopyroxene, same grain as $(6,7,8)$ & 21.8 & 60.5 & 36.7 & 11.1 & - & 14.1 & 7.1 & - & 1.23 & 1.29 & 2.07 \\
\hline 6 & Amp & Rim clinopyroxene, same grain as $(5,7,8)$ & 15.4 & 46.0 & 28.2 & 8.5 & - & 12.5 & 5.7 & - & 1.14 & 1.44 & 1.83 \\
\hline 7 & Amp & Rim clinopyroxene, same grain as $(5,6,8)$ & 13.3 & 42.3 & 32.1 & 8.9 & - & 15.6 & 7.2 & - & 0.94 & 1.41 & 1.24 \\
\hline 8 & Amp & Rim clinopyroxene, same grain as $(5,6,7)$ & 1.1 & 7.3 & 13.1 & 5.0 & - & 7.5 & 3.9 & - & 0.14 & 1.27 & 0.19 \\
\hline \multicolumn{14}{|c|}{ 147-894G-12R-5, 50-57 cm (Piece 7B): } \\
\hline 1 & Amp & Groundmass clot, same grain as (2) & 1.4 & 6.8 & 8.0 & 3.5 & - & 5.5 & 2.8 & - & 0.25 & 1.25 & 0.34 \\
\hline 2 & Amp & Groundmass clot, same grain as (1) & 2.5 & 15.0 & 20.6 & 9.5 & - & 21.3 & 9.6 & - & 0.16 & 1.44 & 0.17 \\
\hline 3 & Amp & Groundmass clot & 1.9 & 10.4 & 12.6 & 4.6 & - & 6.8 & 2.9 & - & 0.25 & 1.53 & 0.44 \\
\hline 4 & Amp & Groundmass clot & 2.3 & 9.0 & 8.7 & 3.3 & - & 7.0 & 3.1 & - & 0.44 & 1.48 & 0.51 \\
\hline 5 & Amp & Groundmass clot & 2.6 & 8.9 & 8.9 & 3.7 & $=$ & 6.8 & 3.3 & $=$ & 0.44 & 1.34 & 0.54 \\
\hline 7 & $\mathrm{Cpx}$ & Same grain as ( 8 ) & 2.3 & 11.1 & 11.1 & 6.5 & 1.2 & 10.5 & 6.6 & 6.8 & 0.22 & 1.05 & 0.24 \\
\hline 8 & Cpx & Same grain as (7) & 2.1 & 9.3 & 10.8 & 5.6 & 1.5 & 10.2 & 5.6 & 6.5 & 0.23 & 1.18 & 0.25 \\
\hline \multicolumn{14}{|c|}{ 147-894G-17R-1, 23-27 cm (Piece 6): } \\
\hline 1 & Amp & Rim clinopyroxene; same grain as (2), next to apatite $(9,10)$ & 7.6 & 44.7 & 50.0 & 17.8 & - & 29.2 & 12.4 & - & 0.27 & 1.54 & 0.42 \\
\hline 2 & Amp & Rim clinopyroxene; same grain as (1), next to apatite $(9,10)$ & 38.0 & 220 & 251 & 113 & - & 261 & 126 & - & 0.21 & 1.35 & 0.20 \\
\hline 3 & Amp & Groundmass clot & 4.8 & 31.5 & 40.5 & 14.0 & - & 20.4 & 9.9 & - & 0.22 & 1.35 & 0.33 \\
\hline 4 & Amp & Groundmass clot & 2.3 & 16.3 & 27.7 & 11.1 & - & 16.2 & 8.3 & - & 0.13 & 1.28 & 0.19 \\
\hline 5 & Amp & Rim clinopyroxene, same grain as $(6,7,8)$ & 8.6 & 49.4 & 53.2 & 20.2 & - & 34.6 & 14.6 & - & 0.27 & 1.55 & 0.40 \\
\hline 6 & Amp & Rim clinopyroxene, same grain as $(5,7,8)$ & 4.7 & 23.2 & 26.9 & 11.3 & - & 18.8 & 8.3 & - & 0.26 & 1.47 & 0.38 \\
\hline 7 & Amp & Rim clinopyroxene, same grain as $(5,6,8)$ & 4.6 & 28.7 & 33.3 & 13.5 & - & 26.2 & 12.6 & - & 0.22 & 1.36 & 0.25 \\
\hline 8 & Amp & Rim clinopyroxene, same grain as $(5,6,7)$ & 1.1 & 8.5 & 14.6 & 7.7 & - & 16.6 & 7.1 & - & 0.09 & 1.53 & 0.11 \\
\hline 9 & Apatite & Same grain as $(10)$ & 318 & 1272 & 1295 & 365 & $=$ & 375 & $181^{1}$ & 132 & 0.55 & 1.33 & 1.19 \\
\hline 10 & Apatite & Same grain as (9) & 306 & 1268 & 1326 & 372 & - & 394 & 186 & 141 & 0.52 & 1.36 & 1.11 \\
\hline \multicolumn{14}{|c|}{ 147-894G-17R-1, 81-86 cm (Piece 11): } \\
\hline 1 & Amp & Rim clinopyroxene $(4,5)$, same grain as $(2,3)$ & 0.4 & 3.2 & 4.2 & 3.0 & - & 2.5 & 1.8 & - & 0.09 & 0.89 & 13.22 \\
\hline 2 & Amp & Rim clinopyroxene $(4,5)$, same grain as $(1,3)$ & 0.8 & 3.4 & 3.2 & 2.0 & - & 4.2 & 2.1 & - & 0.25 & 1.32 & 16.51 \\
\hline 3 & Amp & Rim clinopyroxene $(4,5)$, same grain as $(1,2)$ & 0.8 & 4.0 & 4.5 & 1.9 & $\overline{0}$ & 3.9 & 2.0 & - & 0.26 & 1.25 & 16.00 \\
\hline 4 & Cpx & Same grain as (5) & 0.9 & 3.5 & 4.0 & 2.8 & 0.7 & 4.1 & 2.7 & 2.3 & 0.19 & 0.99 & 20.62 \\
\hline 5 & $\mathrm{Cpx}$ & Same grain as (4) & 0.9 & 4.2 & 5.6 & 3.2 & 0.9 & 5.3 & 3.0 & 3.1 & 0.17 & 1.16 & 22.76 \\
\hline 6 & $\mathrm{Cpx}$ & Same grain as $(7,9)$ & 1.2 & 4.5 & 4.8 & 3.5 & 0.8 & 5.8 & 3.8 & 3.6 & 0.23 & 1.00 & 29.41 \\
\hline 7 & $\mathrm{Cpx}$ & Same grain as $(6,9)$ & 1.2 & 4.5 & 4.0 & 2.4 & 0.6 & 5.1 & 2.6 & 2.4 & 0.32 & 1.30 & 21.40 \\
\hline 8 & $\mathrm{Cpx}$ & Same grain as $(6,8)$ & 1.2 & 4.8 & 5.5 & 2.9 & 0.8 & 5.5 & 3.1 & 2.9 & 0.25 & 1.14 & 24.70 \\
\hline 9 & $\mathrm{Cpx}$ & Same grain as (10) & 0.8 & 3.8 & 4.6 & 2.8 & 0.6 & 4.8 & 2.9 & 2.8 & 0.19 & 1.09 & 21.73 \\
\hline 10 & Cpx & Same grain as (9) & 0.8 & 4.1 & 4.7 & 2.7 & 0.8 & 5.0 & 2,6 & 2.7 & 0.20 & 1.28 & 19.67 \\
\hline 14 & Amp & Rim clinopyroxene, same grain as (15) & 0.4 & 2.1 & 3.3 & 1.9 & - & 3.5 & 1.7 & - & 0.12 & 1.36 & 12.27 \\
\hline 15 & Amp & Rim clinopyroxene, same grain as (14) & 0.4 & 2.1 & 4.3 & 2.1 & - & 3.4 & 2.1 & - & 0.12 & 1.07 & 14.89 \\
\hline \multicolumn{14}{|c|}{ 147-894G-18R-1, 2-3 cm (Piece 1): } \\
\hline 1 & Cpx & Same grain as (2) & 0.7 & 2.9 & 4.6 & 2.1 & 0.7 & 5.0 & 3.2 & 2.8 & 0.20 & 1.02 & 0.14 \\
\hline 2 & $\mathrm{Cpx}$ & Same grain as (1) & 0.4 & 2.5 & 3.9 & 2.1 & 0.9 & 4.7 & 2.5 & 2.8 & 0.13 & 1.23 & 0.11 \\
\hline 3 & Amp & Rim clinopyroxene $(4,6)$ & 1.5 & 4.9 & 5.3 & 2.1 & $\bar{x}$ & 1.7 & 1.3 & - & 0.45 & 0.82 & 0.76 \\
\hline 4 & $\mathrm{Cpx}$ & Same grain as (6) & 0.5 & 1.9 & 2.8 & 1.6 & 0.6 & 3.9 & 2.4 & 2.1 & 0.19 & 1.08 & 0.13 \\
\hline 6 & $\mathrm{Cpx}$ & Same grain as (5) & 0.3 & 1.0 & 1.3 & 0.6 & 0.3 & 1.1 & 0.7 & 0.7 & 0.28 & 1.04 & 0.25 \\
\hline 8 & $\mathrm{Cpx}$ & Same grain as (13) & 0.8 & 3.5 & 4.3 & 3.0 & 0.7 & 4.6 & 2.9 & 2.8 & 0.16 & 1.03 & 0.18 \\
\hline 12 & Amp & Rim clinopyroxene $(4,6)$ & 1.5 & 4.5 & 3.8 & 1.3 & - & 1.7 & 1.4 & - & 0.73 & 0.82 & 0.75 \\
\hline 13 & Cpx & Same grain as (8) & 0.6 & 2.4 & 4.1 & 2.7 & 0.4 & 3.6 & 2.5 & 2.1 & 0.13 & 0.95 & 0.15 \\
\hline
\end{tabular}

Notes: Concentrations in parts per million (ppm). Numbers in parentheses indicate analysis number. La/Sm, Dy/Er, and $\mathrm{La} / \mathrm{Er}$ ratios are chondrite-normalized, with chondritic values from Anders and Grevesse (1989). 

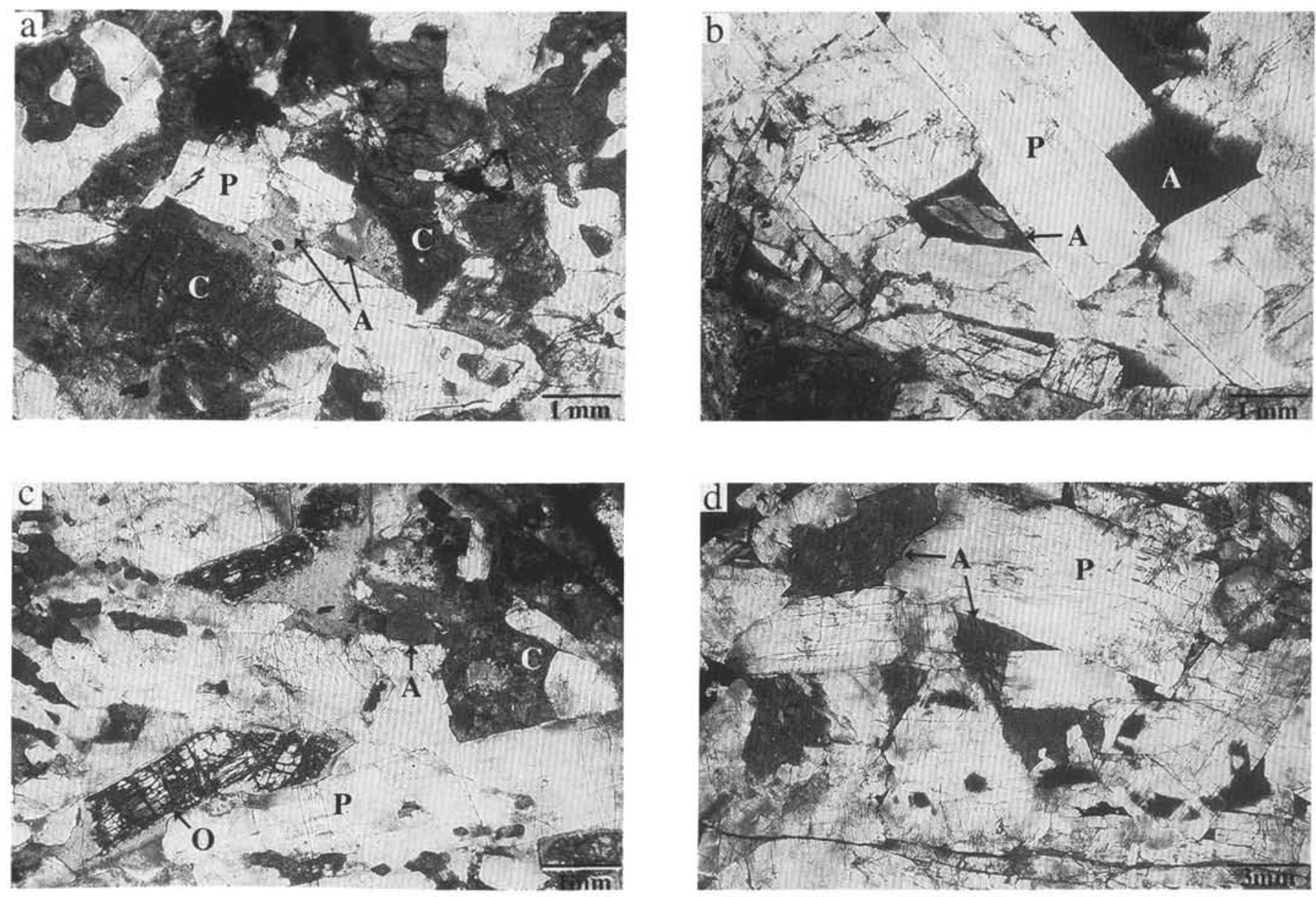

Figure 1. Photomicrographs showing the textures of amphibole analyzed for trace element content. A. Green, granular to fibrous amphibole rim on clinopyroxene in a gabbronorite (Sample 147-894G-17R-1, 81-86 cm, Piece 11). B. Isolated grain of amphibole is intersertal to plagioclase in a gabbronorite (Sample 147894G-12R-5, 50-59 cm, Piece 10). C. Brown amphibole rim on clinopyroxene in a gabbronorite, orthopyroxene is altered to green, fibrous amphibole, and igneous plagioclase is turbid due to minute opaque inclusions (Sample 147-894G-12R-3, 112-115 cm, Piece 7A). D. Randomly oriented, green granular amphibole forms clots that are intersertal to plagioclase in a weakly foliated gabbro (Sample 147-894G-12R-5, 50-57 cm, Piece 7B). C = clinopyroxene, O = orthopyroxene, $\mathrm{P}=$ plagioclase, and $\mathrm{A}=$ amphibole.

Table 3. Representative clinopyroxene analyses, Hole 894G.

\begin{tabular}{|c|c|c|c|c|c|c|c|c|c|c|}
\hline $\begin{array}{l}\text { Core, section: } \\
\text { Interval }(\mathrm{cm}) \text { : } \\
\text { Piece no.: } \\
\text { IP analysis no.: }\end{array}$ & $\begin{array}{c}6 \mathrm{R}-1 \\
112-116 \\
10 \\
3\end{array}$ & $\begin{array}{c}6 \mathrm{R}-1 \\
112-116 \\
10 \\
9\end{array}$ & $\begin{array}{c}8 \mathrm{R}-1 \\
13-15 \\
2 \\
5\end{array}$ & $\begin{array}{c}8 \mathrm{R}-1 \\
13-15 \\
2 \\
2\end{array}$ & $\begin{array}{c}12 R-1 \\
117-125 \\
12 \\
15\end{array}$ & $\begin{array}{c}12 \mathrm{R}-5 \\
50-57 \\
7 \mathrm{~B} \\
8\end{array}$ & $\begin{array}{c}17 \mathrm{R}-1 \\
81-86 \\
11 \\
7\end{array}$ & $\begin{array}{c}17 \mathrm{R}-1 \\
81-86 \\
11 \\
10\end{array}$ & $\begin{array}{c}18 \mathrm{R}-1 \\
2-3 \\
1 \\
4\end{array}$ & $\begin{array}{c}18 \mathrm{R}-1 \\
2-3 \\
1 \\
8\end{array}$ \\
\hline $\begin{array}{l}\mathrm{SiO}_{2} \\
\mathrm{TiO}_{2} \\
\mathrm{Al}_{2} \mathrm{O}_{3} \\
\mathrm{MgO} \\
\mathrm{MnO} \\
\mathrm{FeO}^{\top} \\
\mathrm{CaO}^{\mathrm{T} O} \\
\mathrm{Na}_{2} \mathrm{O} \\
\mathrm{K}_{2} \mathrm{O} \\
\mathrm{Cr}_{2} \mathrm{O}_{3} \\
\mathrm{Total}^{2}\end{array}$ & $\begin{array}{r}52.85 \\
0.21 \\
1.35 \\
12.95 \\
0.20 \\
11.86 \\
20.01 \\
0.25 \\
0.00 \\
0.06 \\
99.75\end{array}$ & $\begin{array}{r}52.70 \\
0.49 \\
1.49 \\
13.34 \\
0.09 \\
10.52 \\
20.52 \\
0.28 \\
0.00 \\
0.34 \\
99.76\end{array}$ & $\begin{array}{r}51.18 \\
0.71 \\
1.96 \\
13.87 \\
0.10 \\
9.66 \\
21.03 \\
0.33 \\
0.00 \\
0.29 \\
99.12\end{array}$ & $\begin{array}{r}52.60 \\
0.71 \\
1.74 \\
14.69 \\
0.12 \\
11.46 \\
18.98 \\
0.30 \\
0.00 \\
0.25 \\
100.84\end{array}$ & $\begin{array}{r}52.20 \\
0.48 \\
1.43 \\
14.75 \\
0.04 \\
10.28 \\
20.70 \\
0.28 \\
0.00 \\
0.28 \\
100.45\end{array}$ & $\begin{array}{r}51.44 \\
0.76 \\
1.84 \\
14.20 \\
0.08 \\
10.94 \\
20.15 \\
0.31 \\
0.00 \\
0.30 \\
100.01\end{array}$ & $\begin{array}{r}52.30 \\
0.62 \\
1.51 \\
13.61 \\
0.31 \\
9.49 \\
22.72 \\
0.22 \\
0.00 \\
0.13 \\
100.90\end{array}$ & $\begin{array}{r}53.15 \\
0.77 \\
1.95 \\
13.72 \\
0.21 \\
10.57 \\
19.99 \\
0.29 \\
0.00 \\
0.15 \\
100.78\end{array}$ & $\begin{array}{r}53.11 \\
0.37 \\
1.02 \\
14.96 \\
0.23 \\
7.16 \\
23.54 \\
0.20 \\
0.00 \\
0.21 \\
100.80\end{array}$ & $\begin{array}{r}52.20 \\
0.64 \\
1.76 \\
14.42 \\
0.19 \\
7.52 \\
23.07 \\
0.28 \\
0.00 \\
0.23 \\
100.30\end{array}$ \\
\hline $\begin{array}{l}\text { Wo } \\
\text { En } \\
\text { Fs } \\
\text { Mg\# }\end{array}$ & $\begin{array}{l}0.39 \\
0.40 \\
0.21 \\
0.66\end{array}$ & $\begin{array}{l}0.40 \\
0.42 \\
0.18 \\
0.69\end{array}$ & $\begin{array}{l}0.42 \\
0.42 \\
0.15 \\
0.72\end{array}$ & $\begin{array}{l}0.37 \\
0.44 \\
0.19 \\
0.70\end{array}$ & $\begin{array}{l}0.41 \\
0.44 \\
0.15 \\
0.72\end{array}$ & $\begin{array}{l}0.40 \\
0.43 \\
0.17 \\
0.70\end{array}$ & $\begin{array}{l}0.45 \\
0.40 \\
0.15 \\
0.72\end{array}$ & $\begin{array}{l}0.38 \\
0.43 \\
0.19 \\
0.70\end{array}$ & $\begin{array}{l}0.46 \\
0.44 \\
0.10 \\
0.79\end{array}$ & $\begin{array}{l}0.46 \\
0.43 \\
0.11 \\
0.77\end{array}$ \\
\hline
\end{tabular}

Notes: Major element composition in weight percent $(w t \%) . \mathrm{FeO}^{\mathrm{T}}=$ total iron as FeO. IP analysis number corresponds to analysis number in Table 2. Wo $=$ wollanstonite, En $=$ enstatite, and Fs = ferrosilite (calculated following the procedure given in Lindsley, 1983, and Lindsley and Anderson, 1983). $\mathrm{Mg} \#=\mathrm{Mg} /\left(\mathrm{Mg}+\mathrm{Fe}{ }^{2+}\right.$ ). 

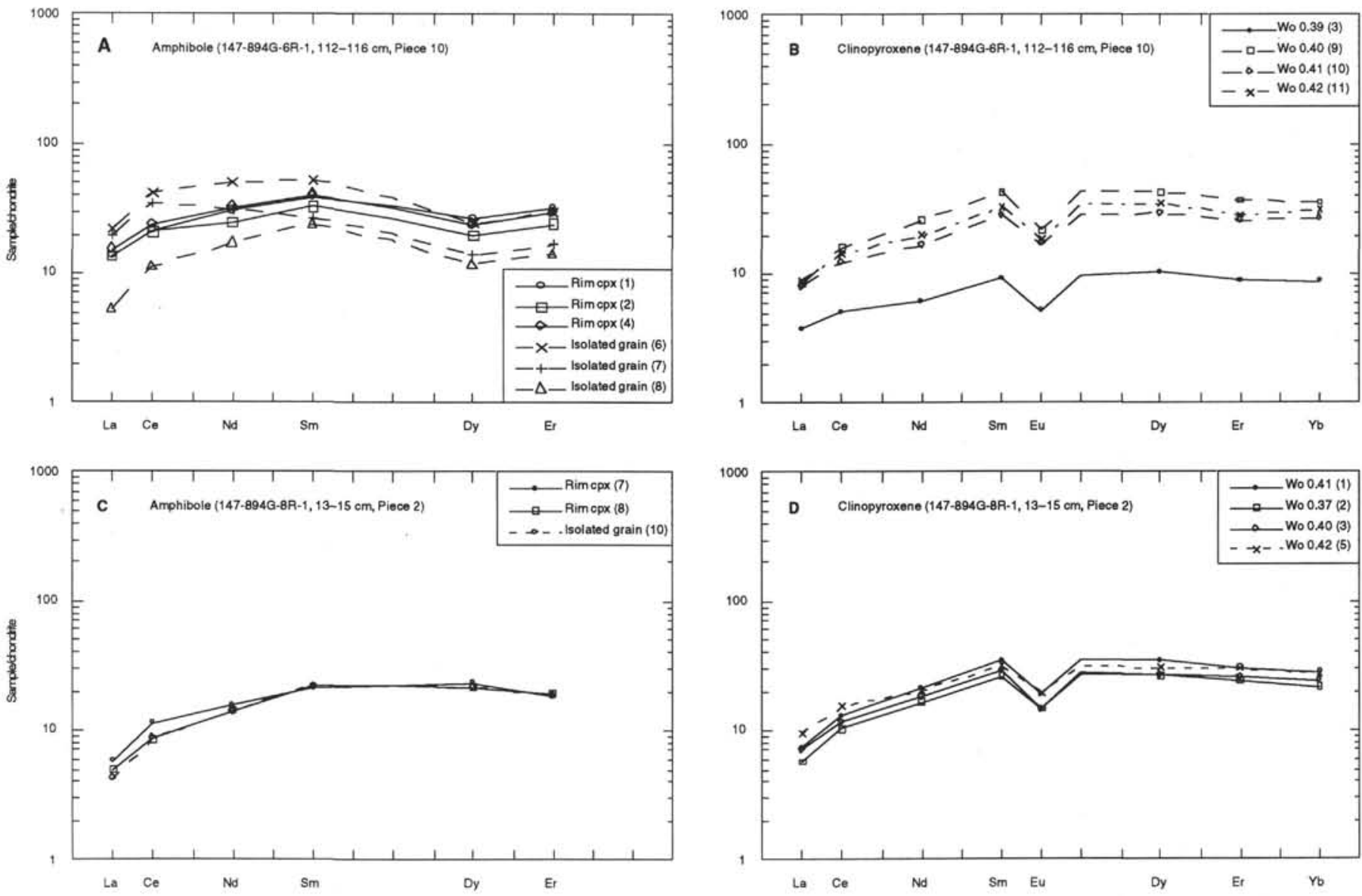

Figure 2. Chondrite-normalized REE profiles of amphibole (A, C, E, F, G, I, J, L, M, O) and clinopyroxene (B, D, H, K, N, P). The occurrence of amphibole is followed by the analysis number (in parentheses); the average wollastonite content of clinopyroxene is given in mol\%, followed by the analysis number (in parentheses). In all plots, each analysis has a separate symbol and analyses of the same grain have the same line. Note that Eu and $\mathrm{Yb}$ contents were not determined for amphibole. Concentrations are normalized to $\mathrm{C} 1$ chondritic values using the elemental abundances of Anders and Grevesse $(1989)$. $\mathrm{cpx}=\mathrm{clinopyrox}$ ene, $\mathrm{gm}=$ groundmass, and $\mathrm{Wo}=$ wollastonite content.

less enriched than the grain intergrown with Fe-Ti oxides (Fig. 2F). The grain surrounded by plagioclase is less aluminous than the grain mixed with Fe-Ti oxides and has a higher Mg\#. In Sample 147-894G$12 \mathrm{R}-3,112-115 \mathrm{~cm}$ (Piece $7 \mathrm{~A}$ ), two grains in a groundmass clot (analyses 3 and 4) have similar REE contents to the amphibole rimming clinopyroxene (analysis 8; Fig. 2I).

Amphibole that rims clinopyroxene has either a granular or fibrous texture and may have a range in REE content. For example, fibrous green amphibole in Sample 147-894G-17R-1, 81-86 cm (Piece 11) (analyses 1-3 and 14-15) has a uniform REE composition whereas brownish green amphibole in Sample 147-894G-12R-1, 117-125 $\mathrm{cm}$ (Piece 12D), is zoned with both light-REE-depleted and -enriched compositions and a broad range in concentration (cf. analyses 1-6 and 16).

As will be discussed in a later section, the presence of an accessory phase(s) may influence the trace element content of amphibole. Apatite occurs adjacent to or within amphibole grains in Samples 147-894G-8R-1, 13-15 cm (Piece 2) (analyses 7 and 8), 12R-1, 112$117 \mathrm{~cm}$ (Piece 12D) (analyses 10-13), and 17R-1, 23-25 cm (Piece 6) (analyses 1 and 2) (note that the analysis number of amphibole adjacent to apatite is given in parentheses). Amphibole grains in contact with apatite are both light-REE-depleted and -enriched, with a range in concentration. Amphibole adjacent to zircon in Sample 147-894G9R-3, 70-76 cm (Piece 5C) (analyses 11, 14, and 15) has chondritenormalized profiles similar to clinopyroxene.

\section{Accessory Phases}

\section{Zircon}

Zircon is a minor phase $(<1$ modal\%) in several intervals in Hole $894 \mathrm{G}$, occurring primarily in coarse-grained gabbronorites where it is associated with Fe-Ti oxides and amphibole. In Sample 147-894G9R-3, 70-76 cm (Piece 5C), zircon is depleted in light REE and significantly enriched in heavy REE, with $(\mathrm{La} / \mathrm{Sm})_{\mathrm{N}}$ and $(\mathrm{Dy} / \mathrm{Er})_{\mathrm{N}}$ ratios from 0.01 to 0.06 and 0.36 to 0.51 , respectively (Fig. 5A).

\section{Apatite}

Apatite is a common minor phase associated with amphibole and $\mathrm{Fe}$-Ti oxides. It is $\mathrm{Cl}$-rich and $\mathrm{F}$-poor, with $\mathrm{Cl}$ and $\mathrm{F}$ contents from 1.3 to 5.8 and $<0.2$ to $0.5 \mathrm{wt} \%$, respectively. All grains have a concavedown REE pattern; $(\mathrm{La} / \mathrm{Sm})_{\mathrm{N}}$ and $(\mathrm{Dy} / \mathrm{Er})_{\mathrm{N}}$ ratios are 0.52 to 0.84 and from 1.33 to 1.80 , respectively (Fig. 5B).

\section{Discussion}

The major element composition of amphibole in the Hole $894 \mathrm{G}$ gabbroic rocks indicate a metamorphic origin. The coexistence of low- and high-Al amphibole in many samples suggests formation at temperatures below the actinolite-hornblende immiscibility gap, at greenschist $\left(250^{\circ}-450^{\circ} \mathrm{C}\right)$ to the transition to the amphibolite facies 

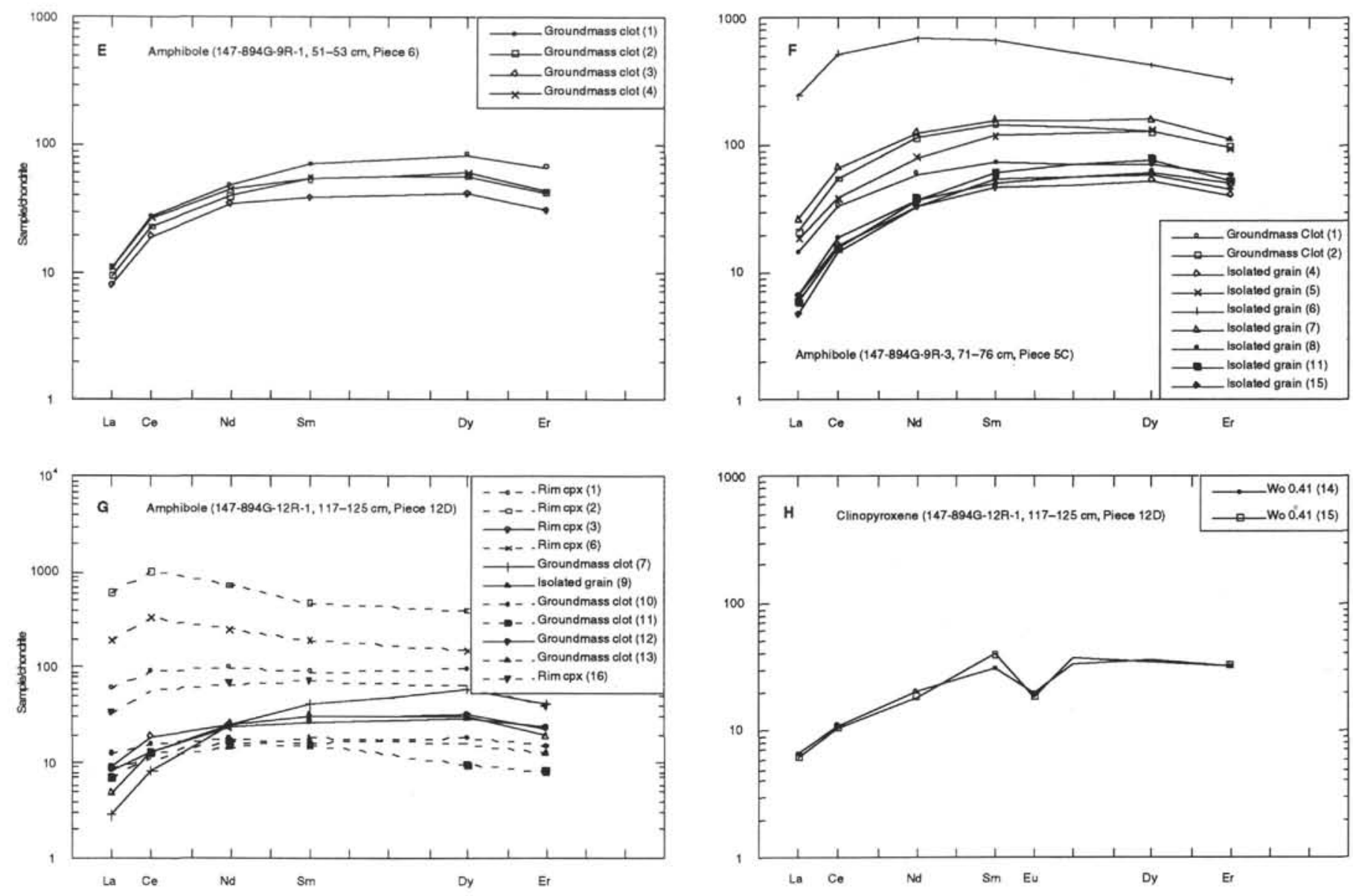

Figure 2 (continued).

$\left(450^{\circ}-600^{\circ} \mathrm{C}\right)$ conditions (Liou et al., 1974; Spear, 1980; Moody et al., 1983). By contrast, granular amphibole that is intersertal to plagioclase and rims clinopyroxene indicate an igneous origin, having crystallized from a melt or exsolved magmatic fluids. In the following sections, these interpretations for the formation of amphibole are evaluated using the REE data. The rationale for this approach is that the REEs are known to be relatively immobile at greenschist to amphibole facies conditions (e.g., Thompson, 1983), implying that igneous amphibole could retain its magmatic REE signature during interaction with seawater-derived hydrothermal fluids.

\section{Role of Seawater-derived Hydrothermal Fluids}

Hydrothermal fluids venting at the seafloor today have distinctive REE profiles, with light REE/heavy REE ratios $>10$ and large positive Eu anomalies (e.g., Campbell et al., 1988). It is likely, however, that these hydrothermal fluids bear little resemblance to fluids deep in the crust. Seawater-derived hydrothermal fluids that reach $>2 \mathrm{~km}$ depth below the seafloor must be very rock-dominated as field and theoretical studies predict very low permeability in plutonic sequences at subsolidus conditions (see summary in Rosenberg et al., 1993). Because the REE content of seawater is several orders of magnitude lower than oceanic crustal material (Elderfield and Greaves, 1982), seawater-derived hydrothermal fluids cannot be more REE-enriched than the rock through which it is passing. The partitioning of the REEs between amphibole and hydrothermal fluids ( $D^{\text {amp-hydrothermal }}$ fluids) has not been determined. Early experimental studies suggest that $\mathrm{D}^{\text {clinopyroxene-fluid }}$ are less than those for $\mathrm{D}^{\text {clinopyroxene-melt }}$ (e.g., Cullers et al., 1973). These conclusions may have no bearing on $D^{\text {amp-hydrothermal }}$ fluids, however, as they were conducted with distilled water and the transport of the REEs in aqueous fluids is strongly influenced by fluid composition and, in particular, the presence of $\mathrm{Cl}$ and $\mathrm{F}$ (Humphris, 1984). If it is assumed that partition coefficients for amphibolehydrothermal fluids are similar to those of hornblende-melt (e.g., Sisson, 1994) and that the REE content of seawater-derived hydrothermal fluids are less than or equal to rock values, it would not be possible to produce REE enrichment greater than rock values. Based on these arguments, amphibole grains with REE contents equal to or less than rock values can be considered hydrothermal in origin. These grains include all textural types and span the range in major element composition of Hole 894G amphibole. Amphibole veins from gabbroic rocks from the Mid-Atlantic Ridge that have the same major element composition as the Hole $894 \mathrm{G}$ amphibole have REE contents less than clinopyroxene but with similar profiles (K.M. Gillis, unpubl. data, 1993). Thus, it is likely that amphibole with the lowest REE contents precipitated from a seawater-derived hydrothermal fluid.

\section{Role of Melt Composition and Accessory Phases}

Hornblende may crystallize from melts that range from basalt to rhyolite in composition (Wones and Gilbert, 1982). For example, an experimental study predicts that amphibole coexists with melts at pressures $\geq 2 \mathrm{~kb}$ where $\mathrm{P}_{\mathrm{H} 2 \mathrm{O}}$ is $\geq 0.6 \mathrm{~kb}$ and temperatures are between $850^{\circ} \mathrm{C}$ and $950^{\circ} \mathrm{C}$ (Spulber and Rutherford, 1983). Such studies also predict that melts become $\mathrm{SiO}_{2}$-rich at high degrees of crystallization (i.e., $<30 \%$ melt), due to the crystallization of Fe-Ti oxides and, to a lesser degree, amphibole (Dixon and Rutherford, 1979; Spulber and Rutherford, 1983). At higher fluid pressure and oxygen fugacity, the upper stability limit of amphibole increases (e.g., Holloway and Burnham, 1972). 

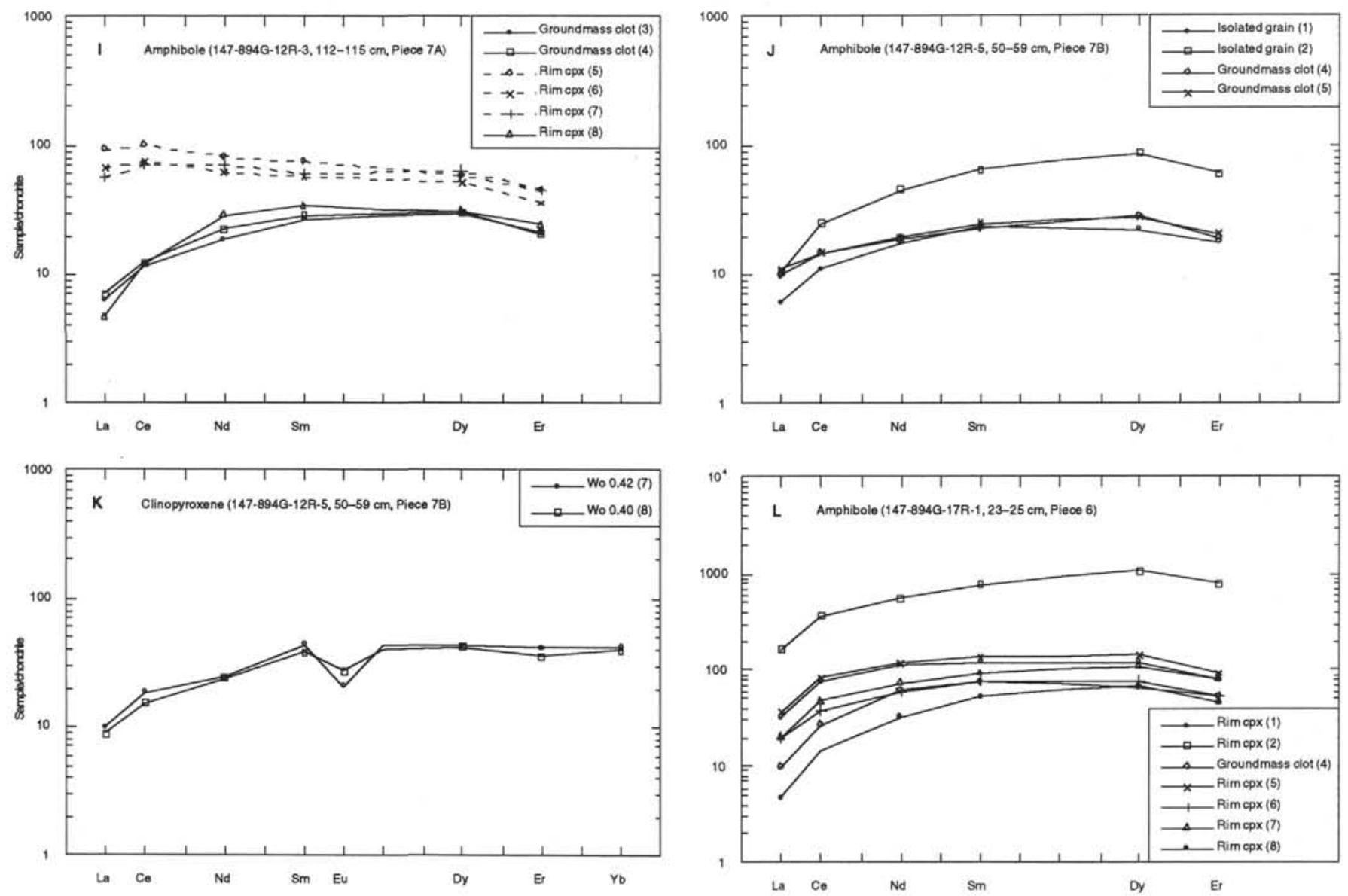

Figure 2 (continued).

The partitioning of the REEs between amphibole and melt is dependent upon magma and mineral composition, pressure, and temperature (Green and Pearson, 1985; Adam et al., 1993; Sisson, 1994), although it has been suggested that magma composition has the most profound effect (Adam et al., 1993). Apparent distribution coefficients (Ds) for the REE between amphibole-melt ( $\left.\mathrm{D}^{\text {amp-melt}}\right)$ increase $\sim 10$ times and become increasingly more convex over a compositional range of basalt to high-silica rhyolite (Sisson, 1994).

Amphibole in the Hole $894 \mathrm{G}$ gabbroic rocks has a broad range in REE contents (Fig. 2). Some samples show a narrow range in REE content whereas other samples show a broad range in REE concentrations (up to 1,000 times chondritic values) with $(\mathrm{La} / \mathrm{Sm})_{\mathrm{N}}$ ratios from 0.1 to 1.4 (Fig. 2). Although there is no systematic relationship between the REE content and texture of amphibole, the most lightREE-enriched amphibole is restricted to granular rims on clinopyroxene that occur within the most chemically evolved intervals. To evaluate whether these variations may be explained by magmatic processes, the REE content of melts in equilibrium with amphibole has been calculated for a range of melt compositions using the apparent Ds of Sisson (1994) (Fig. 6A, B). A comparison of Figures 6A and $6 \mathrm{~B}$ shows that basaltic andesite melts would have higher total REE contents, lower $(\mathrm{La} / \mathrm{Sm})_{\mathrm{N}}$ ratios, and similar $(\mathrm{Dy} / \mathrm{Er})_{\mathrm{N}}$ than dacite melts in equilibrium with the same amphibole grain. These calculations suggest that amphibole crystallized from melts with progressively higher REE contents; in some samples melts evolved without significant fractionation of the REE (e.g., Sample 147-894G-9R-1, $71-76 \mathrm{~cm}$, Piece 5C), whereas in other samples melts became lightREE-enriched (e.g., Sample 147-894G-12R-1, 117-125 cm, Piece 12D). An increase in the total abundance of the REEs is consistent with the crystallization of plagioclase, clinopyroxene, and orthopy- roxene from progressively more evolved melt (e.g., Pedersen and Malpas, 1984). The calculated melts with light-REE-enriched profiles, however, cannot be produced from simple crystallization of the primary igneous phases.

Crystallization of accessory phases will have a profound effect on melt compositions as they strongly partition selective trace elements (Watson, 1980; Watson and Green, 1981; Irving and Frey, 1984). The partitioning of the REEs between apatite and melt is dependent primarily upon magma composition and temperature and is independent of pressure and $\mathrm{H}_{2} \mathrm{O}$ content of the melt (Watson and Green, 1981). In the more evolved intervals of the Hole $894 \mathrm{G}$ core, apatite occurs as a minor phase where it is intergrown with amphibole, $\mathrm{Fe}-\mathrm{Ti}$ oxides, and, less commonly, zircon. Using the apatite REE contents from Hole 894G and Datite-melt determined by Watson and Green (1981), basaltic andesite and andesite melts in equilibrium with Hole $894 \mathrm{G}$ apatite were calculated. These melts are similar to the calculated basaltic andesite and dacite melts in equilibrium with amphibole that have the highest REE content, however, the light REE/heavy REE ratios of the melts in equilibrium with apatite are lower than those in equilibrium with amphibole (Fig. 6). Model calculations predict that the crystallization of apatite in trace amounts (e.g., $\sim 0.1$ modal\%) would prevent further increase in the REE contents and would increase the $(\mathrm{La} / \mathrm{Sm})_{N}$ ratio of a melt (Pedersen and Malpas, 1984; Perfit et al., 1983). Thus, it is unlikely that apatite crystallization could produce the most light-REE-enriched melts in equilibrium with amphibole.

Zircon occurs as an accessory phase associated with Fe-Ti oxides in the most evolved gabbroic intervals and probably postdates amphibole crystallization. Precipitation of zircon will enrich and deplete the light and heavy REE content of a melt, respectively, and fraction- 

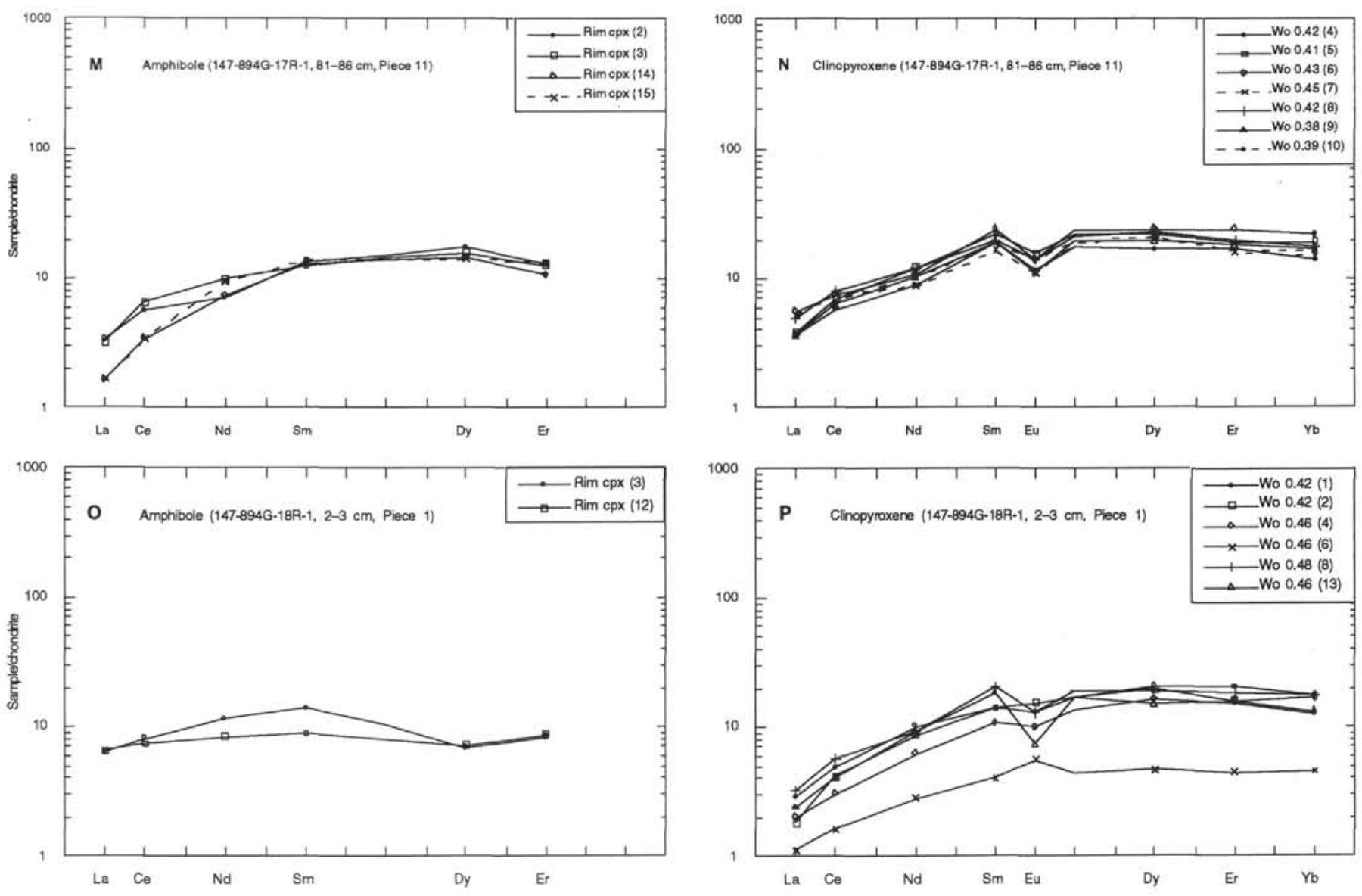

Figure 2 (continued).

ate a melt such that both $(\mathrm{La} / \mathrm{Sm})_{\mathrm{N}}$ and $(\mathrm{Dy} / \mathrm{Er})_{\mathrm{N}}$ ratios would increase (Fig. 5B) (Watson, 1980). The impact of zircon crystallization on melt REE contents, however, would be limited by the $\mathrm{Zr}$ content of the melt.

Calculated melts in equilibrium with amphibole and apatite overlap the field of the most REE-enriched EPR basalts and are significantly more enriched in total REE contents than the Hole $894 \mathrm{G}$ gabbroic rocks (cf. Figs. 6, 7). An exception to this relationship is a coarse-grained, oxide gabbronorite interval in Section 147-894G-9R3 , which is enriched in total REE content and light REEs, has a large negative Eu anomaly, and is depleted in heavy REE (Fig. 7; Pedersen et al., this volume). This evolved interval is similar to other fractionated plutonics from the Hess Deep scarps (J. Natland, pers. comm, 1992) and ODP Hole 735B (J. Hertogen, pers. comm., 1993) which are enriched in total REE content and have light-REE-depleted or -enriched profiles (Fig. 7). For comparison, ophiolitic plagiogranites are enriched in REE and have flat to slightly light-REE-enriched profiles (e.g., Aldiss, 1981; Pedersen and Malpas, 1984). These evolved rock types, however, are not as REE enriched as the most fractionated calculated melts in equilibrium with amphibole and apatite.

Textural and compositions of plagioclase, clinopyroxene, orthopyroxene, and olivine indicate that primitive basaltic melts became moderately fractionated during the initial stages of crystallization, followed by migration of highly fractionated interstitial melts through the cumulus pile (Natland and Dick, this volume). Model calculations show that these interstitial melts were ferroandesites with enriched incompatible element contents (Natland and Dick, this volume; Pedersen et al., this volume). Although these predictions are consistent with the calculated melts, the basaltic andesite and dacite melts with light-REE-enriched profiles have higher total REE contents and are more light-REE-enriched than any known melt composition. Evidence for these light-REE-enriched melts is restricted to most evolved gabbronoritic samples where the light-REE-enriched amphibole rims clinopyroxene. The high REE contents cannot be explained by melt content because $\mathrm{D}^{\text {amp-melt }}$ increases as melts become more siliceous (Sission, 1994). Moreover, it is unlikely that apatite crystallization could produce these compositions as melts in equilibrium with apatite are not as enriched in light REEs or total REE content as the most fractionated melts in equilibrium with amphibole.

\section{Role of Magmatic Fluids}

Amphibole may also crystallize from exsolved magmatic fluids. Melts with mid-ocean ridge basalt compositions only reach $\mathrm{H}_{2} \mathrm{O}$ saturation at high degrees of crystallization $(\sim 95 \%)$, due to the incompatible nature of $\mathrm{H}_{2} \mathrm{O}$ and low $\mathrm{H}_{2} \mathrm{O}$ content of the melt (Burham, 1979). Fluid inclusion data for the Hole $894 \mathrm{G}$ gabbroic rocks studies show that $\mathrm{H}_{2} \mathrm{O}-\mathrm{CO}_{2}-\mathrm{NaCl}- \pm \mathrm{Fe}$-rich fluids were exsolved from evolved interstitial melts (Kelley and Malpas, this volume). Evidence for exsolved fluids is restricted to coarse-grained patches that have the most evolved primary mineral compositions (Kelley and Malpas, this volume), similar to the occurrences of the light-REE-enriched amphibole. If amphibole crystallized from fluids with compositions similar to those indicated by the fluid inclusions, the light-REE-enriched amphibole may be explained by complexing with $\mathrm{Cl}^{-}$as the light REE are strongly complexed with $\mathrm{Cl}^{-}$at high temperatures (Valsami-Jones et al., 1994). As magmatic fluids were probably exsolved at temperatures between $700^{\circ} \mathrm{C}$ and $800^{\circ} \mathrm{C}$ (Wyllie, 1977; Burnham, 
Table 4. Average representative amphibole analyses, Hole 894G.

\begin{tabular}{|c|c|c|c|c|c|c|c|c|c|c|c|c|c|c|c|}
\hline $\begin{array}{l}\text { Core, section: } \\
\text { Interval (cm): } \\
\text { Piece no.: } \\
\text { IP analysis no.: } \\
\text { Occurrence: }\end{array}$ & $\begin{array}{c}6 \mathrm{R}-1 \\
112-116 \\
10 \\
2 \\
\text { Rim cpx }\end{array}$ & $\begin{array}{c}6 \mathrm{R}-1 \\
112-116 \\
10 \\
8(3) \\
\text { Is grain }\end{array}$ & $\begin{array}{c}8 \mathrm{R}-1 \\
13-15 \\
2 \\
7(2) \\
\text { Rim cpx }\end{array}$ & $\begin{array}{c}9 \mathrm{R}-3 \\
51-54 \\
4 \\
3(3) \\
\text { Gm clot }\end{array}$ & $\begin{array}{c}12 \mathrm{R}-\mathrm{I} \\
117-125 \\
12 \mathrm{~d} \\
2(2) \\
\text { Rim cpx }\end{array}$ & $\begin{array}{c}12 \mathrm{R}-1 \\
117-125 \\
12 \mathrm{~d} \\
2(2) \\
\text { Rim cpx }\end{array}$ & $\begin{array}{c}12 R-1 \\
117-125 \\
12 \mathrm{~d} \\
11(3) \\
\mathrm{Gm} \text { clot }\end{array}$ & $\begin{array}{c}12 R-3 \\
112-115 \\
7 \mathrm{a} \\
4(4) \\
\text { Gm clot }\end{array}$ & $\begin{array}{c}12 R-3 \\
112-115 \\
7 a \\
7(3) \\
\text { Rim cpx }\end{array}$ & $\begin{array}{c}12 R-3 \\
112-115 \\
7 a \\
8(3) \\
\text { Rim cpx }\end{array}$ & $\begin{array}{c}12 \mathrm{R}-5 \\
50-57 \\
7 \mathrm{~b} \\
1(2) \\
\text { Is grain }\end{array}$ & $\begin{array}{c}12 \mathrm{R}-5 \\
50-57 \\
7 \mathrm{~b} \\
2(4) \\
\text { Is grain }\end{array}$ & $\begin{array}{c}17 \mathrm{R}-1 \\
23-27 \\
6 \\
5 \\
\text { Rim cpx }\end{array}$ & $\begin{array}{c}17 \mathrm{R}-1 \\
23-27 \\
6 \\
8(3) \\
\text { Rim cpx }\end{array}$ & $\begin{array}{c}18 \mathrm{R}-1 \\
2-3 \\
1 \\
12(2) \\
\text { Rim cpx }\end{array}$ \\
\hline $\begin{array}{l}\mathrm{SiO}_{2} \\
\mathrm{TiO}_{2} \\
\mathrm{Al}_{2} \mathrm{O}_{3} \\
\mathrm{Cr}_{2} \mathrm{O}_{3} \\
\mathrm{FeO}^{\mathrm{T}} \\
\mathrm{MnO} \\
\mathrm{MgO} \\
\mathrm{CaO} \\
\mathrm{Na}_{2} \mathrm{O} \\
\mathrm{K}_{2} \mathrm{O} \\
\mathrm{Cl} \\
\mathrm{F} \\
\text { Total }\end{array}$ & $\begin{array}{r}50.84 \\
1.06 \\
4.78 \\
0.06 \\
13.67 \\
0.11 \\
15.32 \\
11.52 \\
0.90 \\
0.26 \\
0.20 \\
0.25 \\
98.97\end{array}$ & $\begin{array}{r}50.83 \\
0.34 \\
4.33 \\
0.06 \\
16.67 \\
0.36 \\
15.16 \\
10.49 \\
0.81 \\
0.19 \\
0.21 \\
0.25 \\
99.70\end{array}$ & $\begin{array}{r}52.37 \\
0.65 \\
3.75 \\
0.02 \\
12.21 \\
0.14 \\
16.92 \\
11.97 \\
0.61 \\
0.09 \\
0.11 \\
0.15 \\
98.98\end{array}$ & $\begin{array}{r}53.48 \\
0.40 \\
1.17 \\
0.02 \\
16.51 \\
0.28 \\
14.96 \\
10.73 \\
0.25 \\
0.04 \\
- \\
\overline{9} .84\end{array}$ & $\begin{array}{r}55.63 \\
0.19 \\
1.06 \\
0.07 \\
12.14 \\
0.28 \\
17.19 \\
12.21 \\
0.15 \\
0.01 \\
- \\
\overline{-}\end{array}$ & $\begin{array}{r}49.85 \\
1.29 \\
5.77 \\
0.11 \\
16.10 \\
0.26 \\
12.97 \\
11.58 \\
0.99 \\
0.32 \\
\overline{-} \\
99.25\end{array}$ & $\begin{array}{r}55.17 \\
0.29 \\
1.50 \\
0.10 \\
11.79 \\
0.31 \\
18.21 \\
11.71 \\
0.21 \\
0.05 \\
0.07 \\
0.13 \\
99.52\end{array}$ & $\begin{array}{r}50.55 \\
0.58 \\
4.65 \\
0.09 \\
15.47 \\
0.26 \\
13.78 \\
11.98 \\
0.61 \\
0.12 \\
0.19 \\
0.11 \\
98.41\end{array}$ & $\begin{array}{r}48.02 \\
1.41 \\
6.64 \\
0.07 \\
14.17 \\
0.23 \\
14.43 \\
11.62 \\
1.21 \\
0.50 \\
0.28 \\
0.14 \\
98.71\end{array}$ & $\begin{array}{r}54.82 \\
0.14 \\
1.45 \\
0.04 \\
12.47 \\
0.19 \\
17.05 \\
12.15 \\
0.22 \\
0.04 \\
0.07 \\
0.07 \\
98.72\end{array}$ & $\begin{array}{r}50.64 \\
0.37 \\
4.64 \\
0.01 \\
16.03 \\
0.26 \\
13.94 \\
11.61 \\
0.75 \\
0.10 \\
0.10 \\
0.26 \\
98.72\end{array}$ & $\begin{array}{r}52.89 \\
0.41 \\
2.61 \\
0.02 \\
15.55 \\
0.22 \\
15.85 \\
10.47 \\
0.53 \\
0.08 \\
0.17 \\
0.26 \\
99.04\end{array}$ & $\begin{array}{r}50.33 \\
1.01 \\
3.91 \\
0.02 \\
17.04 \\
0.23 \\
13.83 \\
10.58 \\
0.77 \\
0.37 \\
\overline{-} \\
98.10\end{array}$ & $\begin{array}{r}53.53 \\
0.24 \\
1.84 \\
0.03 \\
15.80 \\
0.25 \\
14.74 \\
11.15 \\
0.37 \\
0.06 \\
- \\
\overline{98.01}\end{array}$ & $\begin{array}{r}47.71 \\
0.73 \\
7.90 \\
0.10 \\
14.60 \\
0.06 \\
14.35 \\
12.06 \\
1.67 \\
0.10 \\
0.27 \\
0.30 \\
99.85\end{array}$ \\
\hline \multicolumn{16}{|c|}{ Cation proportions on the basis of 23 anhydrous $\mathrm{O}$} \\
\hline $\begin{array}{l}\mathrm{Si} \\
\mathrm{Al}^{\mathrm{IV}} \\
\mathrm{Al}^{\mathrm{VI}} \\
\mathrm{Cr} \\
\mathrm{Fe}^{3+} \\
\mathrm{Fe}^{2+} \\
\mathrm{Mn} \\
\mathrm{Mg} \\
\mathrm{Ti} \\
\mathrm{Ca} \\
\mathrm{Na} \\
\mathrm{K} \\
\mathrm{Cl} \\
\mathrm{F} \\
\mathrm{Mg}\end{array}$ & $\begin{array}{l}7.33 \\
0.67 \\
0.14 \\
0.01 \\
0 \\
1.65 \\
0.01 \\
3.29 \\
0.12 \\
1.78 \\
0.25 \\
0.05 \\
0.05 \\
0.11 \\
0.67\end{array}$ & $\begin{array}{l}7.31 \\
0.64 \\
0.10 \\
0.01 \\
0.31 \\
1.70 \\
0.04 \\
3.25 \\
0.04 \\
1.62 \\
0.23 \\
0.03 \\
0.03 \\
0.08 \\
0.66\end{array}$ & $\begin{array}{l}7.41 \\
0.55 \\
0.07 \\
0.00 \\
0.30 \\
1.14 \\
0.01 \\
3.57 \\
0.07 \\
1.82 \\
0.17 \\
0.02 \\
0.03 \\
0.07 \\
0.76\end{array}$ & $\begin{array}{l}7.79 \\
0.18 \\
0.02 \\
0.00 \\
0.06 \\
1.95 \\
0.03 \\
3.25 \\
0.04 \\
1.67 \\
0.07 \\
0.01 \\
0.00 \\
0.00 \\
0.62\end{array}$ & $\begin{array}{l}7.86 \\
0.13 \\
0.05 \\
0.01 \\
0.05 \\
1.39 \\
0.03 \\
3.62 \\
0.02 \\
1.85 \\
0.04 \\
0.00 \\
0.00 \\
0.00 \\
0.72\end{array}$ & $\begin{array}{l}7.20 \\
0.80 \\
0.18 \\
0.01 \\
0.29 \\
1.65 \\
0.02 \\
2.79 \\
0.14 \\
1.79 \\
0.28 \\
0.06 \\
0.00 \\
0.00 \\
0.63\end{array}$ & $\begin{array}{l}7.73 \\
0.25 \\
0.00 \\
0.01 \\
0.15 \\
1.23 \\
0.04 \\
3.80 \\
0.03 \\
1.76 \\
0.06 \\
0.01 \\
0.02 \\
0.06 \\
0.76\end{array}$ & $\begin{array}{l}7.33 \\
0.67 \\
0.12 \\
0.01 \\
0.33 \\
1.55 \\
0.02 \\
2.98 \\
0.06 \\
1.86 \\
0.18 \\
0.02 \\
0.05 \\
0.05 \\
0.66\end{array}$ & $\begin{array}{l}6.95 \\
1.05 \\
0.08 \\
0.01 \\
0.43 \\
1.28 \\
0.01 \\
3.11 \\
0.15 \\
1.80 \\
0.33 \\
0.09 \\
0.07 \\
0.06 \\
0.71\end{array}$ & $\begin{array}{l}7.78 \\
0.22 \\
0.02 \\
0.00 \\
0.10 \\
1.39 \\
0.02 \\
3.61 \\
0.02 \\
1.85 \\
0.06 \\
0.01 \\
0.02 \\
0.03 \\
0.72\end{array}$ & $\begin{array}{l}7.33 \\
0.67 \\
0.12 \\
0.00 \\
0.34 \\
1.60 \\
0.02 \\
3.01 \\
0.04 \\
1.80 \\
0.21 \\
0.02 \\
0.03 \\
0.12 \\
0.65\end{array}$ & $\begin{array}{l}7.61 \\
0.39 \\
0.05 \\
0.00 \\
0.09 \\
1.77 \\
0.03 \\
3.40 \\
0.04 \\
1.61 \\
0.15 \\
0.01 \\
0.04 \\
0.12 \\
0.66\end{array}$ & $\begin{array}{l}7.39 \\
0.61 \\
0.07 \\
0.00 \\
0.02 \\
2.08 \\
0.03 \\
3.03 \\
0.11 \\
1.67 \\
0.22 \\
0.07 \\
0.00 \\
0.00 \\
0.59\end{array}$ & $\begin{array}{l}7.78 \\
0.22 \\
0.09 \\
0.00 \\
0.05 \\
1.86 \\
0.03 \\
3.19 \\
0.03 \\
1.74 \\
0.10 \\
0.01 \\
0.00 \\
0.00 \\
0.63\end{array}$ & $\begin{array}{l}6.83 \\
1.17 \\
0.16 \\
0.01 \\
0.51 \\
1.24 \\
0.00 \\
3.06 \\
0.08 \\
1.85 \\
0.46 \\
0.02 \\
0.07 \\
0.13 \\
0.71\end{array}$ \\
\hline
\end{tabular}

Notes: Major element compositions in weight $\%$. FeO ${ }^{\mathrm{T}}=$ total iron as $\mathrm{FeO}$. IP analysis number corresponds to analysis number in Table 2. Number in parentheses $=$ number of analyses. Compositions were recalculated from microprobe data on the basis of 23 anhydrous oxygens. $\mathrm{Fe}^{3+}$ was calculated as the average of the maximum and minimum ferric iron content for solutions that satisfied charge balance constraints, assigning Ca to the $\mathrm{M} 4$ site and $\mathrm{K}$ to Site $\mathrm{A}$ (Robinson et al., 1982). $\mathrm{Mg} \#=\mathrm{Mg} /\left(\mathrm{Mg}+\mathrm{Fe}^{2+}\right)$. Rim $\mathrm{cpx}=$ rim clinopyroxene, Is grain $=$ isolated grain, and $\mathrm{Gm}$ clot $=$ groundmass clot. 

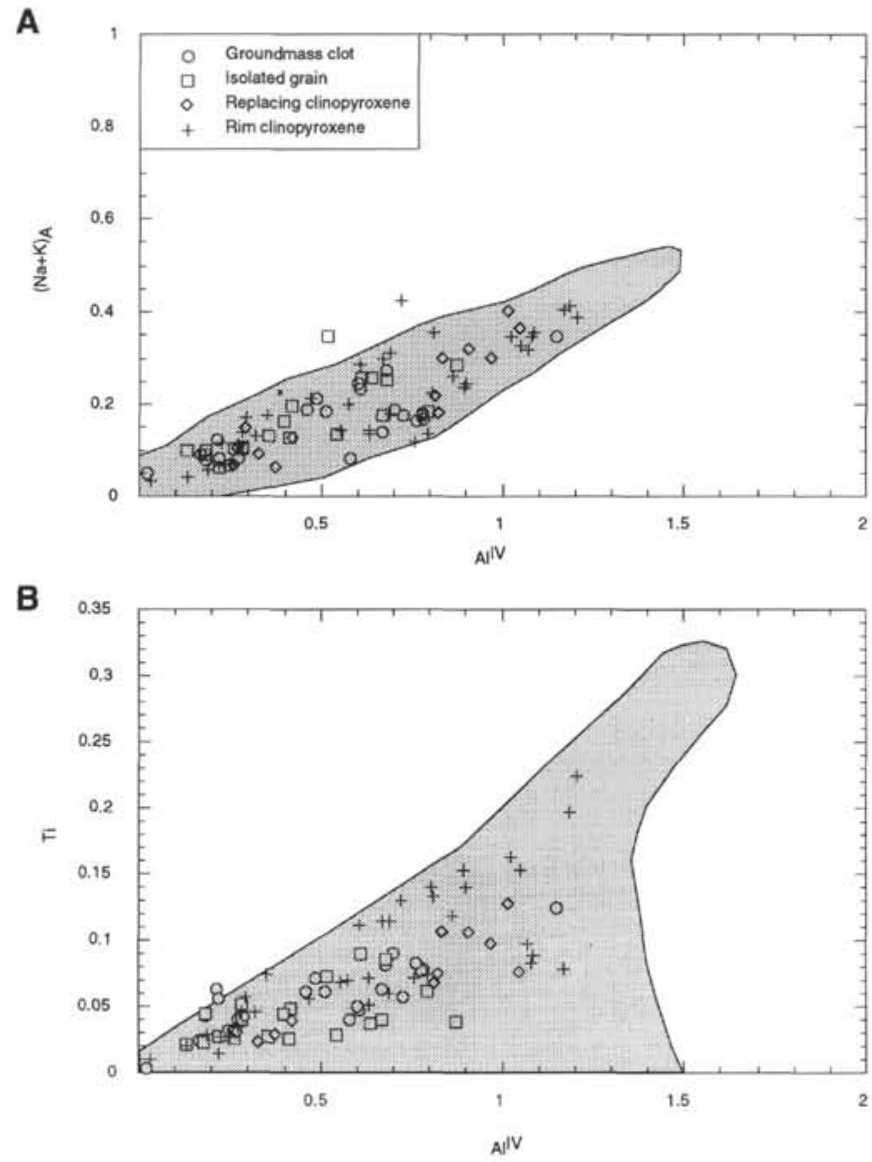

Figure 3. Composition of amphibole in the samples analyzed for trace elements. A. Al ${ }^{\mathrm{IV}}$ vs. $(\mathrm{Na}+\mathrm{K})$ in Site A. B. Al ${ }^{\mathrm{IV}}$ vs. Ti content. The shaded area represents the range in composition of amphibole from other Hole 894G and Hess Deep samples (K.M. Gillis, unpubl. data).

1979; Kelley and Malpas, this volume), they cannot account for the major element content of amphibole. Fluid inclusion data show, however, that exsolved fluids migrated along microfractures and were not entrapped until they had cooled to $\sim 300^{\circ} \mathrm{C}$ (Kelley and Malpas, this volume). Thus, it is plausible that amphibole crystallized from exsolved magmatic fluids that had cooled to $300^{\circ}-600^{\circ} \mathrm{C}$. Although it is not possible to evaluate what proportion of the igneous amphibole formed from magmatic fluids, it is the most viable explanation for the light-REE-enriched amphibole.

\section{SUMMARY}

Textural and compositional data indicate that groundmass amphibole in the Hole $894 \mathrm{G}$ gabbroic rocks record a complex history of crystallization that spans both igneous and metamorphic processes. The major element composition of amphibole indicates a metamorphic origin whereas textural relations indicate both an igneous and metamorphic origin. The REE data show no systematic variation with either major element composition or textural type; however, amphibole with the highest REE contents form as rims on clinopyroxene. Amphibole grains within an individual sample may have REE contents less than or equal to clinopyroxene, show a continuous increase in REE content with no fractionation, or become light-REEenriched with increasing REE content. The origin of amphibole with REE contents less than or equal to clinopyroxene cannot be distinguished by compositional criteria. By contrast, amphibole with REE

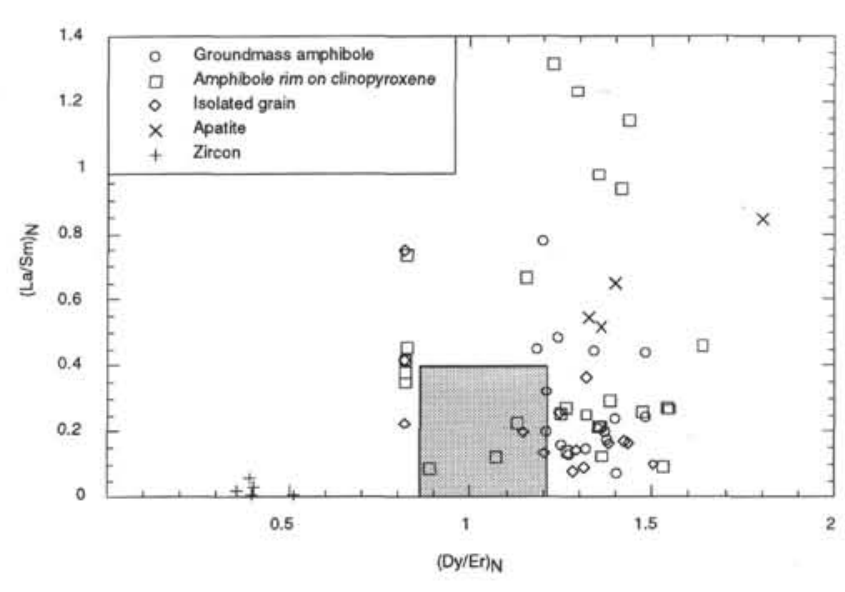

Figure 4. $(\mathrm{Dy} / \mathrm{Er})_{\mathrm{N}} \mathrm{vs} .(\mathrm{La} / \mathrm{Sm})_{\mathrm{N}}$ of amphibole. The shaded area indicates the range in composition of clinopyroxene.

contents greater than clinopyroxene could not have formed by interaction with seawater-derived hydrothermal fluids and require an igneous origin. This would require that the major element composition of igneous amphibole was reset by interaction with seawater-derived hydrothermal fluids at subsolidus temperatures.

Textural and geochemical data for all igneous phases in the Hole $894 \mathrm{G}$ gabbroic rocks indicate that evolved interstitial melts migrated through the cumulus pile during the latest stages of crystallization (Natland and Dick, this volume; Pedersen et al., this volume). Calculated melt compositions in equilibrium with igneous amphibole show that the REE content of late-stage melts progressively increased and that the light REEs were locally enriched. Calculated melt compositions in equilibrium with apatite overlap the upper range of calculated melts in equilibrium amphibole but have lower light REE/heavy REE ratios. Crystallization of apatite would increase the $(\mathrm{La} / \mathrm{Sm})_{\mathrm{N}}$ ratio of a melt but could not produce the most fractionated melts. Enrichment in the total REE content is consistent with the crystallization of plagioclase and the pyroxenes and slight increases in light REE content may reflect crystallization of trace apatite and zircon. These trends are consistent with known compositions of evolved oceanic plutonics, however, they do not account for the light-REE-enriched melts. Alternatively, it is plausible that amphibole crystallized from magmatic fluids that were exsolved from a melt during the final stages of crystallization. Fluid inclusion data show that $\mathrm{H}_{2} \mathrm{O}-\mathrm{CO}_{2}-\mathrm{NaCl}- \pm \mathrm{Fe}-$ rich fluids were present in the most evolved intervals of the Hole 894G core (Kelley and Malpas, this volume), where light-REE-enriched amphibole has been identified. As these fluids were trapped at temperatures between $300^{\circ} \mathrm{C}$ and $600^{\circ} \mathrm{C}$, it may be predicted that amphibole crystallized from cooled magmatic fluids, thus accounting for the major element composition of amphibole. Based on the results of this and other studies of the Hole $894 \mathrm{G}$ core, there was a continuum between magmatic and hydrothermal processes. Groundmass amphibole records a complex history of crystallization from an evolved melt and its exsolved magmatic fluids, followed by interaction with seawater-derived hydrothermal fluids.

\section{ACKNOWLEDGMENTS}

I would like to thank the many colleagues and friends who contributed to this paper. The Sedco and ODP crews of JOIDES Resolution, C. Mével, my co-chief scientist, and J. Allan, our staff scientist, made Leg 147 memorable and successful. Discussions with H. Dick, J. Malpas, R. Pedersen, D. Kelley, N. Shimizu, J. Natland, and, in particular, P. Meyer were invaluable. N. Shimizu and K. Burrhus provided guidance in the collection of the trace element data; H. Dick, J. 
Malpas, R. Pedersen, D. Kelley, and C. Manning shared samples and/ or data; N. Chatterjee assisted with the microprobe analyses at MIT; and K. Sapp provided support in many aspects of this project. T. Sisson is gratefully acknowledged for providing a preprint with the working curves and distribution coefficients that were used in this paper.

\section{REFERENCES}

Adam, J., Green, T.H., and Sie, S.H., 1993. Proton microprobe determined partitioning of $\mathrm{Rb}, \mathrm{Sr}, \mathrm{Ba}, \mathrm{Y}, \mathrm{Zr}, \mathrm{Nb}$, and $\mathrm{Ta}$ between experimentally produced amphiboles and silicate melts with variable F content. Chem. Geol., 109:29-49.

Aldiss, D.T., 1981. Plagiogranites from the ocean crust and ophiolites. $\mathrm{Na}$ ture, 289:577-578.

Anders, E., and Grevesse, N., 1989. Abundances of the elements: meteoritic and solar. Geochim. Cosmochim. Acta, 53:197-214.

Burnham, C.W., 1979. Magma and hydrothermal fluids. In Barnes, H.L. (Ed.), Geochemistry of Hydrothermal Ore Deposits: New York (Wiley), 71-133.

Campbell, A.C., Palmer, M.R., Klinkhammer, G.P., Bowers, T.S., Edmond, J.M., Lawrence, J.R., Casey, J.F., Thompson, G., Humphris, S., Rona, P.A., and Karson, J.A., 1988. Chemistry of hot springs on the Mid-Atlantic Ridge. Nature, 335:514-519.

Dixon, S., and Rutherford, M.J., 1979. Plagiogranites as late-stage immiscible liquids in ophiolite and mid-ocean ridge suites: an experimental study. Earth Planet. Sci. Lett., 45:45-60.

Elderfield, H., and Greaves, M.J., 1982. The rare earth elements in seawater. Nature, 296:214-219.

Gillis, K.M., Thompson, G., and Kelley, D.S., 1993. A view of the lower crustal component of hydrothermal systems at the Mid-Atlantic Ridge. $J$. Geophys. Res., 98:19597-19619.

Grandjean, P., 1989. Les terres rares et la composition isotopique du neodyme dans les phosphates biogenes: tracers des processus paleooceanographiques et sedimentaires [Ph.D. thesis]. Univ. Nancy, France.

Green, T.H., and Pearson, N.J., 1985. Experimental determination of REE partition coefficients between amphibole and basaltic to andesitic liquids at high pressure. Geochim. Cosmochim. Acta, 49:1465-1468.

Holloway, J.R., and Burnham, C.W., 1972. Melting relations of basalt with equilibrium water pressure less than total pressure. J. Petrol., 13:1-29.

Humphris, S., 1984. The mobility of rare earth elements in the crust. In Henderson, P. (Ed.), Rare Earth Element Geochemistry: Amsterdam (Elsevier), 317-342.

Irving, A.J., and Frey, F.A., 1984. Trace element abundances in megacrysts and their host basalt: constraints on partition coefficients and megacryst genesis. Geochim. Cosmochim. Acta, 48:1201-1221.

Johnson, K.T.M., and Dick, H.J.B., 1992. Open system melting and temporal and spatial variation of peridotite and basalt at the Atlantis II Fracture Zone. J. Geophys. Res., 97:9219-9241.

Johnson, K.T.M., Dick, H.J.B., and Shimizu, N., 1990. Melting in the oceanic upper mantle: an ion microprobe study of diopsides in abyssal peridotites. J. Geophys. Res., 95:2661-2678.

Leake, B.E., 1978. Nomenclature of amphiboles. Am. Mineral., 63:10231052.

Lindsley, D.H., 1983. Pyroxene thermometry. Am. Mineral., 68:477-493.

Lindsley, D.H., and Andersen, D.J., 1983. A two-pyroxene thermometer. $J$. Geophys. Res., 88(Suppl.):A887-A906.

Liou, J.G., Kuniyoshi, S., and Ito, K., 1974. Experimental studies of the phase relations between greenschist and amphibolite in a basaltic system. Am. J. Sci., 274:613-632.

Manning, C.E., and Bird, D.K., 1986. Hydrothermal clinopyroxenes of the Skaergaard intrusion. Contrib. Mineral. Petrol., 92:437-447.

Mével, C., 1987. Evolution of oceanic gabbros from DSDP Leg 82: influence of the fluid phase on metamorphic crystallizations. Earth Planet. Sci. Lett., 83:67-79.

Mével, C., and Cannat, M., 1991. Lithospheric stretching and hydrothermal processes in oceanic gabbros from slow-spreading ridges. In Peters, T.,
Nicolas, A., and Coleman, R.J. (Eds.), Ophiolite Genesis and Evolution of the Oceanic Lithosphere. Petrol. Struct. Geol., 5:293-312.

Meyer, P.S., and Shibata, T., 1990. Complex zoning in plagioclase feldspars from ODP Site 648. In Detrick, R., Honnorez, J., Bryan, W.B., Juteau, T., et al., Proc. ODP, Sci. Results, 106/109: College Station, TX (Ocean Drilling Program), 123-142.

Moody, J.B., Meyer, D., and Jenkins, J.E., 1983. Experimental characterization of the greenschist/amphibolite boundary in mafic systems. Am. J. Sci., 283:48-92.

Pedersen, R.B., 1986. The nature and significance of magma chamber margins in ophiolites: examples from the Norwegian Caledonides. Earth Planet. Sci. Lett., 77:100-112.

Pedersen, R.B., and Malpas, J., 1984. The origin of oceanic plagiogranites from the Karmoy Ophiolite, western Norway. Contrib. Mineral. Petrol., $88: 36-52$.

Perfit, M.R., Fornari, D.J., Malahoff, A., and Embley, R.W., 1983. Geochemical studies of abyssal lavas recovered by DSRV Alvin from eastern Galapagos Rift, Inca Transform and Ecuador Rift, 3. Trace element abundances and petrogenesis. J. Geophys. Res., 88:10551-10572.

Robinson, P., Spear, F.S., Schumacher, J.C., Laird, J., Klein, C., Evans, B.W., and Doolan, B.L., 1982. Phase relations of metamorphic amphiboles: natural occurrence and theory. In Veblen, D.R., and Ribbe, P.H. (Eds.), Amphiboles: Petrology and Experimental Phase Relations. Rev. Mineral., 9B:1-227.

Rosenberg, N.D., Spera, F.J., and Haymon, R.M., 1993. The relationship between flow and permeability field in seafloor hydrothermal systems. Earth Planet. Sci. Lett., 116:135-153.

Shimizu, N., and Le Roex, A.P., 1986. The chemical zoning of augite phenocrysts in alkaline basalts from Gough Island, South Atlantic. J. Volcanol. Geotherm. Res., 29:159-188.

Shipboard Scientific Party, 1993. Site 894. In Gillis, K., Mével, C., Allan, J., et al., Proc. ODP, Init. Repts., 147: College Station, TX (Ocean Drilling Program), 45-108.

Sisson, T.W., 1994. Hornblende-melt trace-element partitioning measured by ion microprobe. Chem. Geol., 117:331-344.

Spear, F.S., 1980. NaSi-CaAl exchange equilibrium between plagioclase and amphibole: an empirical model. Contrib. Mineral. Petrol., 72:33-41.

Spulber, S.D., and Rutherford, M.J., 1983. The origin of rhyolite and plagiogranite in oceanic crust: an experimental study. J. Petrol., 24:1-25.

Stakes, D., Mével, C., Cannat, M., and Chaput, T., 1991. Metamorphic stratigraphy of Hole 735B. In Von Herzen, R.P., Robinson, P.T., et al., Proc. ODP, Sci. Results, 118: College Station, TX (Ocean Drilling Program), 153-180.

Thompson, G., 1983. Hydrothermal fluxes in the Ocean. In Riley, J.P., and Chester, R. (Eds.), Chemical Oceanography, 8: London (Acad. Press), 271-337.

Valsami-Jones, E., Ragnarsdottir, V., Haas, J., and Bailey, E.H., 1994. Rare earth element transport in crustal fluids. Eos, 75:718.

Von Damm, K.L., 1990. Seafloor hydrothermal activity: black smoker chemistry and chimneys. Annu. Rev. Earth Planet. Sci., 18:173-204.

Watson, E.B., 1980. Some experimentally determined zircon/liquid partition coefficients for the rare earth elements. Geochim. Cosmochim. Acta, 44:895-897.

Watson, E.B., and Green, T.H., 1981. Apatite/liquid partition coefficients for the rare earth elements and strontium. Earth Planet. Sci. Lett., 56:405421 .

Wones, D.R., and Gilbert, M.C., 1982. Amphiboles in the igneous environment: introduction. In Veblen, D.R., and Ribbe, P.H. (Eds.), Amphiboles: Petrology and Experimental Phase Relations. Rev. Mineral., 9B:355390.

Wyllie, P.J., 1977. Crustal anatexis: an experimental review. Tectonophysics, 43:41-71.

Date of initial receipt: 25 August 1994

Date of acceptance: 2 March 1995

Ms 147SR-012 

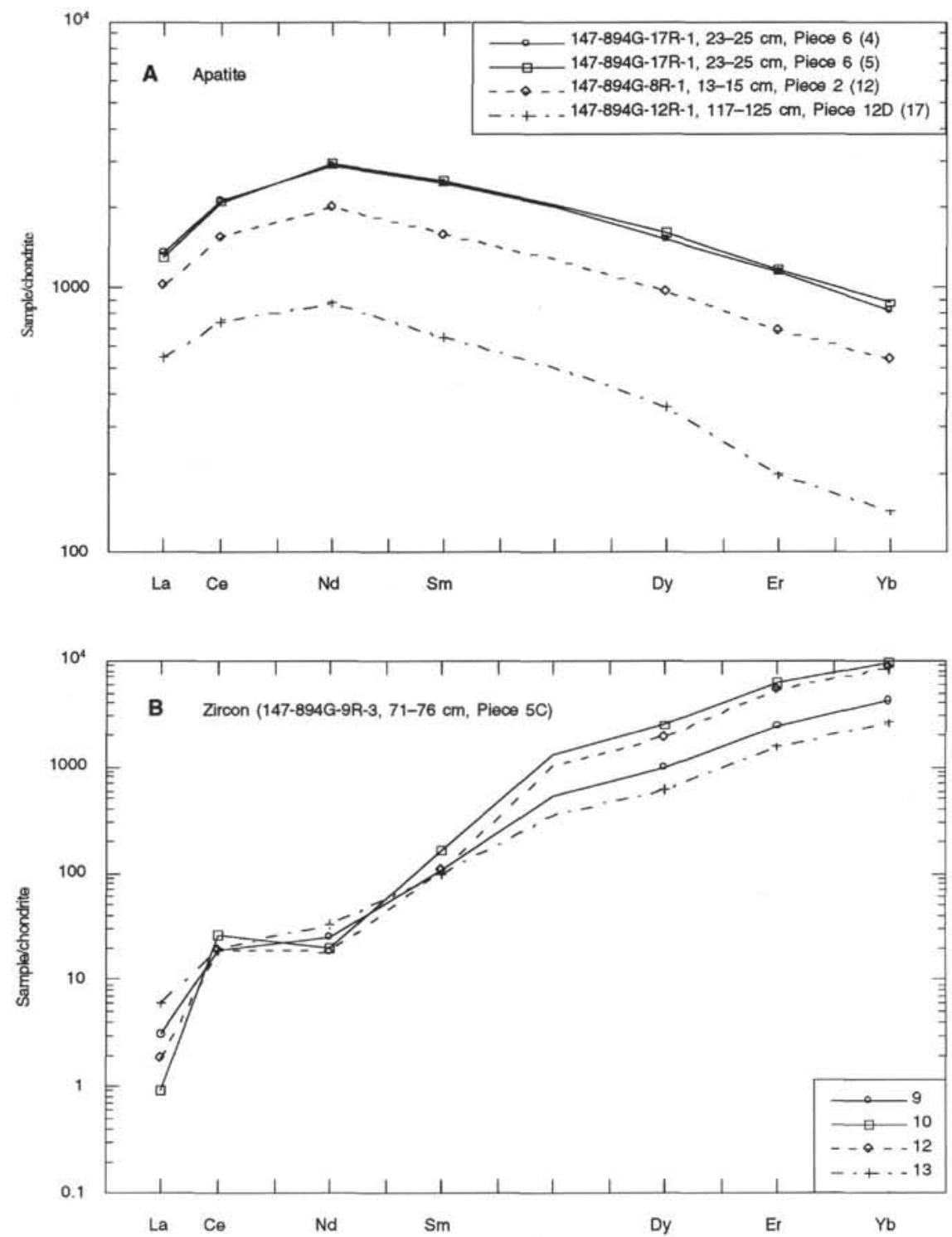

Figure 5. Chondrite-normalized REE profiles of the accessory phases apatite and zircon. Analysis numbers are listed in parentheses. 

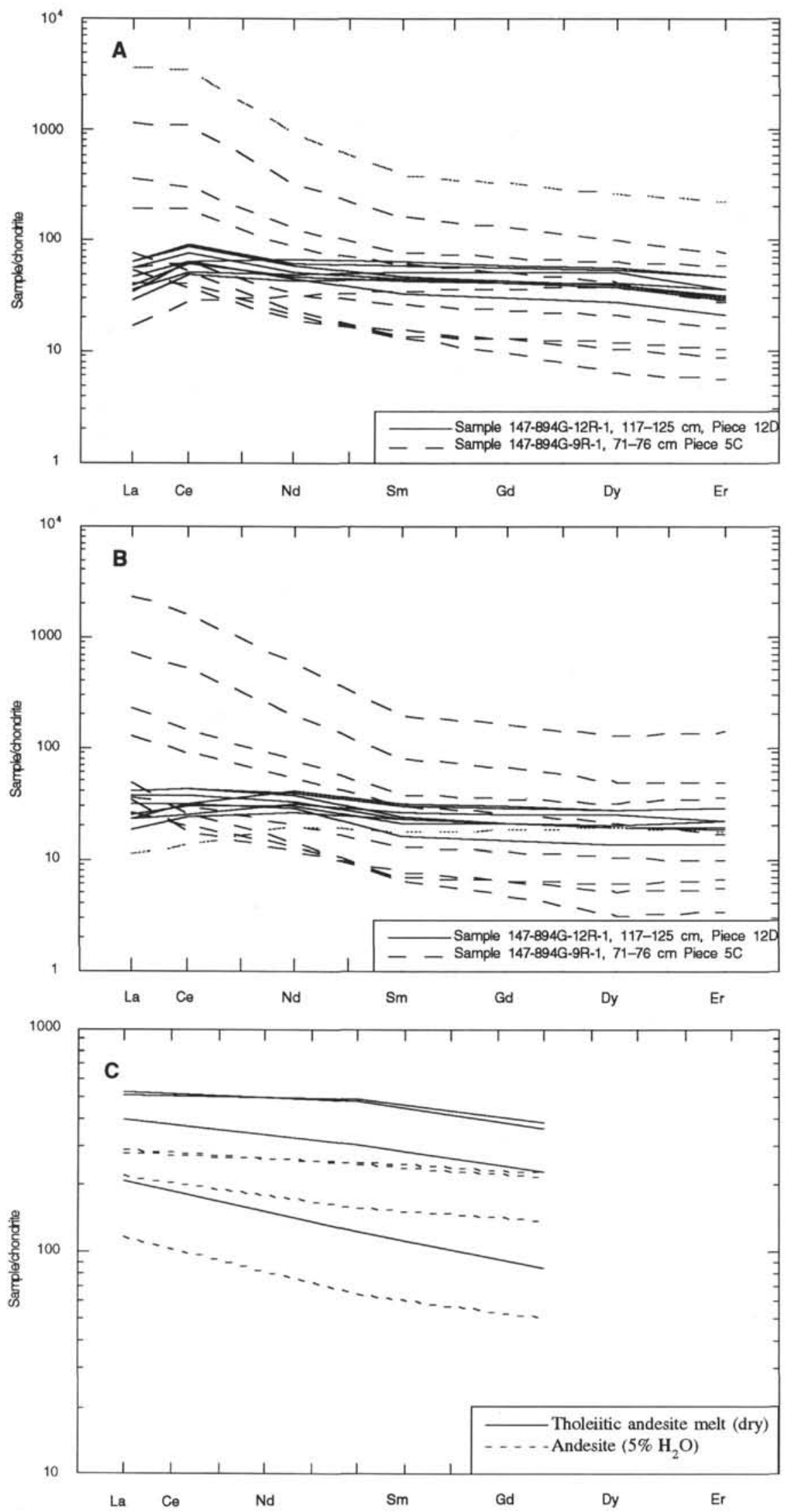

Figure 6. Calculated melt compositions in equilibrium with amphibole from Samples 147-894G-9R-1, 71-76 cm (Piece 5C), and 12R-1, 117-125 cm (Piece 12D). A. Basaltic andesite melts in equilibrium with amphibole. B. Dacite melts in equilibrium with amphibole. C. Calculated melt compositions in equilibrium with apatite from Samples 147-894G-8R-1, 12-15 cm (Piece 2), 12R-1, 117-125 cm (Piece 12D), and 17R-1, 23-25 cm (Piece 6). 




Figure 7. Chondrite-normalized REE profiles of bulk rocks showing the range in composition of gabbronorites from Hole $894 \mathrm{G}$ (Pedersen et al., this volume), EPR volcanics (N. Blum, pers. comm, 1992), and felsic veins associated with oceanic plutonics (tonalite vein from J. Natland, pers. comm, 1992; and trondhjemite vein from J. Hertogen, pers. comm, 1993).

APPENDIX

Proportion of Phases and Average Mineral Compositions for Each Ion Microprobe Analysis

\begin{tabular}{|c|c|c|c|c|c|c|c|}
\hline $\begin{array}{c}\text { Analysis } \\
\text { no. }\end{array}$ & Mineral & Comments & Phases & $\begin{array}{l}\text { Cpx } \\
\text { (Wo) }\end{array}$ & $\begin{array}{c}\mathrm{Cpx} \\
(\mathrm{Mg} \#)\end{array}$ & $\begin{array}{l}\text { Amp } \\
\left(\mathrm{AI}^{\mathrm{IV}}\right)\end{array}$ & $\begin{array}{c}\text { Amp } \\
(\mathrm{Mg} \#)\end{array}$ \\
\hline \multicolumn{8}{|c|}{ 147-894G-6R-1, 112-116 cm (Piece 10): } \\
\hline 1 & Amp & $\operatorname{Rim} \operatorname{cpx}(3)$ & $100 \%$ amp & - & - & 0.69 & 0.636 \\
\hline 2 & Amp & $\operatorname{Rim} \operatorname{cpx}(3)$ & $100 \%$ amp & - & - & 0.67 & 0.67 \\
\hline 3 & Cpx & & $30 \%$ amp & 0.39 & 0.66 & - & - \\
\hline 4 & Amp & $\operatorname{Rim} \operatorname{cpx}(3)$ & $100 \%$ amp & - & - & 0.9 & 0.71 \\
\hline 6 & Amp & Isolated grain, same grain as $(7,8)$ & $100 \%$ amp & - & - & 0.42 & 0.65 \\
\hline 7 & Amp & Isolated grain, same grain as $(7,8)$ & $100 \%$ amp & - & - & 0.41 & 0.66 \\
\hline 8 & Amp & Isolated grain, same grain as $(7,9)$ & $100 \%$ amp & - & - & 0.64 & 0.66 \\
\hline 9 & Cpx & Same grain as $(9,10)$ & $<5 \%$ amp & 0.4 & 0.69 & 0.26 & 0.71 \\
\hline 10 & Cpx & Same grain as $(9,11)$ & $100 \% \mathrm{cpx}$ & 0.41 & 0.7 & - & - \\
\hline 11 & Cpx & Same grain as $(9,10)$ & $20 \%$ amp & 0.42 & 0.71 & 0.81 & 0.69 \\
\hline \multicolumn{8}{|c|}{ 147-894G-8R-1, 13-15 cm (Piece 2) } \\
\hline 1 & $\mathrm{Cpx}$ & Same grain as $(2,3)$ & $100 \% \mathrm{cpx}$ & 0.41 & 0.72 & - & - \\
\hline 2 & Cpx & Same grain as $(1,3)$ & $100 \% \mathrm{cpx}$ & 0.37 & 0.7 & - & - \\
\hline 3 & $\mathrm{Cpx}$ & Same grain as $(1,2)$ & $<5 \%$ amp & 0.4 & 0.71 & - & - \\
\hline 5 & Cpx & & $<5 \%$ amp & 0.42 & 0.72 & 1.19 & 0.66 \\
\hline 7 & Amp & Rim cpx, same grain as (8), next to apatite (12) & $30 \%$ high- $\mathrm{Al}$ amp; $70 \%$ low- $\mathrm{Al}$ amp & - & - & $1.0 / 0.65$ & $0.68 / 0.76$ \\
\hline 8 & Amp & Rim cpx, same grain as (7), next to apatite (12) & $100 \%$ amp & - & - & 0.57 & 0.71 \\
\hline 10 & Amp & Isolated grain & $100 \%$ amp & - & - & 0.8 & 0.76 \\
\hline 12 & Apatite & Next to amphibole $(7,8)$ & & & & & \\
\hline \multicolumn{8}{|c|}{ 147-894G-9R-3, 51-54 cm (Piece 4): } \\
\hline 1 & Amp & Groundmass clot & $100 \%$ amp & - & - & 0.21 & 0.65 \\
\hline 2 & Amp & Groundmass clot & $100 \%$ amp & - & - & 0.21 & 0.65 \\
\hline 3 & Amp & Groundmass clot & $100 \%$ amp & - & - & 0.18 & 0.63 \\
\hline 4 & Amp & Groundmass clot & $100 \%$ amp & - & - & 0.22 & 0.64 \\
\hline \multicolumn{8}{|c|}{ 147-894G-9R-3, 70-76 cm (Piece 5C): } \\
\hline 1 & Amp & Groundmass clot & $100 \%$ amp & - & - & 0.46 & 0.65 \\
\hline 2 & Amp & Groundmass clot & $100 \%$ amp & - & - & 0.48 & 0.65 \\
\hline 4 & Amp & Isolated grain, same grain as (5) & $100 \%$ amp & - & - & 0.28 & 0.64 \\
\hline 5 & Amp & Isolated grain, same grain as (4) & $100 \%$ amp & - & - & 0.22 & 0.63 \\
\hline 6 & Amp & Isolated grain, same grain as (7) & $100 \%$ amp & - & - & 0.54 & 0.54 \\
\hline 7 & Amp & Isolated grain, same grain as (8) & $100 \%$ amp & - & - & 0.61 & 0.6 \\
\hline 8 & Amp & Isolated grain & $100 \%$ amp & - & - & 0.18 & 0.65 \\
\hline 9 & Zircon & & & & & & \\
\hline 10 & Zircon & & & & & & \\
\hline 11 & Amp & Isolated grain, next to zircon & $100 \%$ amp & - & - & 0.25 & 0.68 \\
\hline 12 & Zircon & & & & & & \\
\hline 13 & Zircon & & & & & & \\
\hline \multicolumn{8}{|c|}{ 147-894G-9R-3, 70-76 cm (Piece 5C): } \\
\hline 14 & Amp & Isolated grain, next to zircon & $100 \%$ amp & - & - & 0.13 & 0.67 \\
\hline 15 & Amp & Isolated grain, next to zircon & $100 \%$ amp & - & - & 0.2 & 0.67 \\
\hline \multicolumn{8}{|c|}{ 147-894G-12R-1, 117-125 cm (Piece 12D): } \\
\hline 1 & Amp & Rim cpx, same grain as $(2,3,6,16)$ & High-Al amp with $>50 \%$ low-Al amp & - & - & & \\
\hline 2 & Amp & Rim cpx, same grain as $(1,3,6,16)$ & High-Al amp with $<10 \%$ low-Al amp & - & - & $0.81 / 0.19$ & $0.61 / 0.64$ \\
\hline 3 & Amp & Rim cpx, same grain as $(1,2,6,16)$ & $50 \%$ high $\mathrm{Al}$-amp, $50 \%$ low $\mathrm{Al}$-amp & - & - & $0.8 / 0.13$ & $0.72 / 0.63$ \\
\hline
\end{tabular}


APPENDIX (continued).

\begin{tabular}{|c|c|c|c|c|c|c|c|}
\hline $\begin{array}{c}\text { Analysis } \\
\text { no. }\end{array}$ & Mineral & Comments & Phases & $\begin{array}{l}\text { Cpx } \\
\text { (Wo) }\end{array}$ & $\begin{array}{c}\text { Cpx } \\
(\mathrm{Mg \#})\end{array}$ & $\begin{array}{l}\text { Amp } \\
\left(A I^{\mathrm{v}}\right)\end{array}$ & $\begin{array}{c}\text { Amp } \\
(\mathrm{Mg} \#)\end{array}$ \\
\hline 6 & Amp & Rim cpx, same grain as $(1,2,316)$ & $100 \%$ amp & - & - & $1.2 / 0.24$ & $0.65 / 0.63$ \\
\hline 7 & Amp & Groundmass clot & $50 \%$ high $\mathrm{Al}$-amp, $50 \%$ low-Al amp & - & - & 0.89 & 0.64 \\
\hline 9 & Amp & Isolated grain & $100 \%$ amp & - & - & $0.6 / 0.02$ & $0.53 / 0.76$ \\
\hline 10 & Amp & Groundmass clot & $100 \%$ amp & - & - & 0.36 & 0.71 \\
\hline 11 & Amp & Groundmass clot & $100 \%$ amp & - & 一 & 0.39 & 0.74 \\
\hline 12 & Amp & Groundmass clot & $100 \%$ amp & - & - & 0.25 & 0.76 \\
\hline 13 & Amp & Groundmass clot & $100 \% \mathrm{amp}$ & - & - & 0.68 & 0.7 \\
\hline 14 & Cpx & Same grain as (15) & $100 \% \mathrm{cpx}$ & 0.41 & 0.72 & 0.73 & 0.64 \\
\hline 15 & Cpx & Same grain as (14) & $100 \%$ срx & 0.41 & 0.72 & - & - \\
\hline 16 & Amp & Rim cpx, same grain as $(1,2,3,6)$ & & & & & \\
\hline 17 & Apatite & & & & & & \\
\hline \multicolumn{8}{|c|}{ 147-894G-12R-3, 112-115 cm (Piece 7A): } \\
\hline 2 & $\mathrm{Cpx}$ & & $100 \% \mathrm{cpx}$ & 0.4 & 0.72 & 0.33 & 0.78 \\
\hline 3 & Amp & Groundmass clot & $100 \%$ amp & - & - & $1.06 / 0.64$ & $0.66 / 0.64$ \\
\hline 4 & Amp & Groundmass clot & $100 \%$ amp & - & - & 0.67 & 0.66 \\
\hline 5 & Amp & Rim cpx, same grain as $(6,7,8)$ & $100 \%$ amp & - & - & 0.86 & 0.74 \\
\hline 6 & Amp & Rim cpx, same grain as $(5,7,8)$ & $100 \%$ amp & - & - & 1.02 & 0.72 \\
\hline 7 & Amp & Rim cpx, same grain as $(5,6,8)$ & $100 \%$ amp & 一 & - & 1.05 & 0.7 \\
\hline 8 & Amp & Rim cpx, same grain as $(5,6,7)$ & $100 \%$ amp & - & - & $1.07 / 022$ & $0.65 / 0.72$ \\
\hline \multicolumn{8}{|c|}{ 147-894G-12R-5, 50-59 cm (Piece 7B): } \\
\hline 1 & Amp & Groundmass clot, same grain as (2) & $70 \%$ high- $\mathrm{Al}$ amp, $30 \%$ low- $\mathrm{Al}$ amp & - & - & $0.67 / 0.28$ & $0.66 / 0.68$ \\
\hline 2 & Amp & Groundmass clot, same grain as (1) & $100 \%$ amp & - & - & 0.39 & 0.66 \\
\hline 3 & Amp & Groundmass clot & $100 \%$ amp & - & - & & \\
\hline 4 & Amp & Groundmass clot & $100 \%$ amp & - & - & 0.78 & 0.67 \\
\hline 5 & Amp & Groundmass clot & $100 \%$ amp & - & - & 0.74 & 0.67 \\
\hline 7 & Cpx & Same grain as (8) & $100 \% \mathrm{cpx}$ & 0.42 & 0.7 & - & - \\
\hline 8 & Cpx & Same grain as (7) & $100 \% \mathrm{cpx}$ & 0.4 & 0.7 & & \\
\hline \multicolumn{8}{|c|}{ 147-894G-17R-1, 23-27 cm (Piece 7): } \\
\hline 1 & Amp & Rim cpx; same grain as (2), next to apatite $(9,10)$ & $100 \%$ amp & - & - & 0.72 & 0.71 \\
\hline 2 & Amp & Rim cpx; same grain as (1), next to apatite $(9,10)$ & $100 \%$ amp & - & - & 0.35 & 0.72 \\
\hline 3 & Amp & Groundmass clot & $100 \%$ amp & - & - & 0.6 & 0.55 \\
\hline \multicolumn{8}{|c|}{ 147-894G-12R-5, 50-59 cm (Piece 7B): } \\
\hline 4 & Amp & Groundmass clot & $<5 \%$ low Al-amp & - & - & $0.3 / 0.03$ & $0.5 / 0.73$ \\
\hline 5 & Amp & Rim cpx, same grain as $(6,7,8)$ & $100 \%$ amp & - & - & 0.37 & 0.61 \\
\hline 6 & Amp & Rim cpx, same grain as $(5,7,8)$ & $50 \%$ high-Al amp, $50 \%$ low-Al amp & - & - & $0.47 / 0.04$ & $0.56 / 0.63$ \\
\hline 7 & Amp & Rim cpx, same grain as $(5,6,8)$ & $100 \%$ amp & 一 & - & 0.28 & 0.66 \\
\hline 8 & Amp & Rim cpx, same grain as $(5,6,7)$ & $100 \%$ amp & - & - & 0.22 & 0.63 \\
\hline 9 & Apatite & Same grain as (10) & & & & & \\
\hline 10 & Apatite & Same grain as (9) & & & & & \\
\hline \multicolumn{8}{|c|}{ 147-894G-17R-1, 81-86 cm (Piece 11): } \\
\hline 1 & Amp & Rim cpx $(4,5)$, same grain as $(2,3)$ & $100 \%$ amp & - & - & 0.79 & 0.67 \\
\hline 2 & Amp & Rim cpx $(4,5)$, same grain as $(1,3)$ & $100 \%$ amp & - & - & 0.69 & 0.69 \\
\hline 3 & Amp & Rim cpx $(4,5)$, same grain as $(1,2)$ & $100 \%$ amp & - & - & 0.76 & 0.66 \\
\hline 4 & Cpx & Same grain as (5) & $20 \%$ amp & 0.42 & 0.72 & 0.42 & 0.84 \\
\hline 5 & $\mathrm{Cpx}$ & Same grain as (4) & $<5 \%$ amp & 0.41 & 0.71 & - & - \\
\hline 6 & Cpx & Same grain as $(7,9)$ & $100 \% \mathrm{cpx}$ & 0.43 & 0.71 & - & - \\
\hline 7 & Cpx & Same grain as $(6,9)$ & $\sim 10 \%$ amp & 0.45 & 0.72 & - & - \\
\hline 8 & Cpx & Same grain as $(6,8)$ & $\sim 10 \%$ amp & 0.42 & 0.71 & - & - \\
\hline 9 & Cpx & Same grain as (10) & $100 \% \mathrm{cpx}$ & 0.38 & 0.71 & - & - \\
\hline 10 & $\mathrm{Cpx}$ & Same grain as (9) & $100 \% \mathrm{cpx}$ & 0.39 & 0.71 & - & - \\
\hline 14 & Amp & Rim cpx, same grain as (15) & $<10 \%$ cpx & 0.47 & 0.75 & 0.46 & 0.67 \\
\hline 15 & Amp & Rim cpx, same grain as (14) & & - & - & 0.63 & 0.72 \\
\hline \multicolumn{8}{|c|}{ 147-894G-18R-1, 2-3 cm (Piece 1): } \\
\hline 1 & Cpx & Same grain as (2) & $<5 \%$ amp & 0.42 & 0.75 & 0.97 & 0.72 \\
\hline 2 & Cpx & Same grain as (1) & $<5 \%$ amp & 0.41 & 0.75 & 0.29 & 0.8 \\
\hline 3 & Amp & $\operatorname{Rim} \operatorname{cpx}(4,6)$ & $100 \%$ amp & - & - & 1.08 & 0.74 \\
\hline 4 & Cpx & Same grain as (6) & $\sim 10 \%$ amp & 0.46 & 0.78 & - & - \\
\hline 6 & Cpx & Same grain as (5) & $100 \% \mathrm{cpx}$ & 0.46 & 0.77 & - & - \\
\hline 8 & Cpx & Same grain as (13) & $100 \%$ cpx & 0.48 & 0.9 & - & - \\
\hline 12 & Amp & Rim cpx $(4,6)$ & $100 \%$ amp & - & - & 1.7 & 0.71 \\
\hline 13 & Cpx & Same grain as (8) & $<20 \%$ amp & 0.46 & 0.8 & 0.83 & 0.75 \\
\hline
\end{tabular}

Notes: Proportions of phases were determined from backscattered electron images. $\mathrm{cpx}=$ clinopyroxene, Wo = wollasonite content in mol\%, and Amp $=$ amphibole. Dash $(-)=$ not present. Wo, $\mathrm{Mg \#}$, and $\mathrm{Al}^{\mathrm{IV}}$ are average values. Average values of high- and low- $\mathrm{Al}$ amphibole are separated by a slash $(/)$. 